\title{
Feature
}

\section{Genomics and Proteomics:}

\section{A Signal \\ Processor's Tour}

\section{P. P. Vaidyanathan}

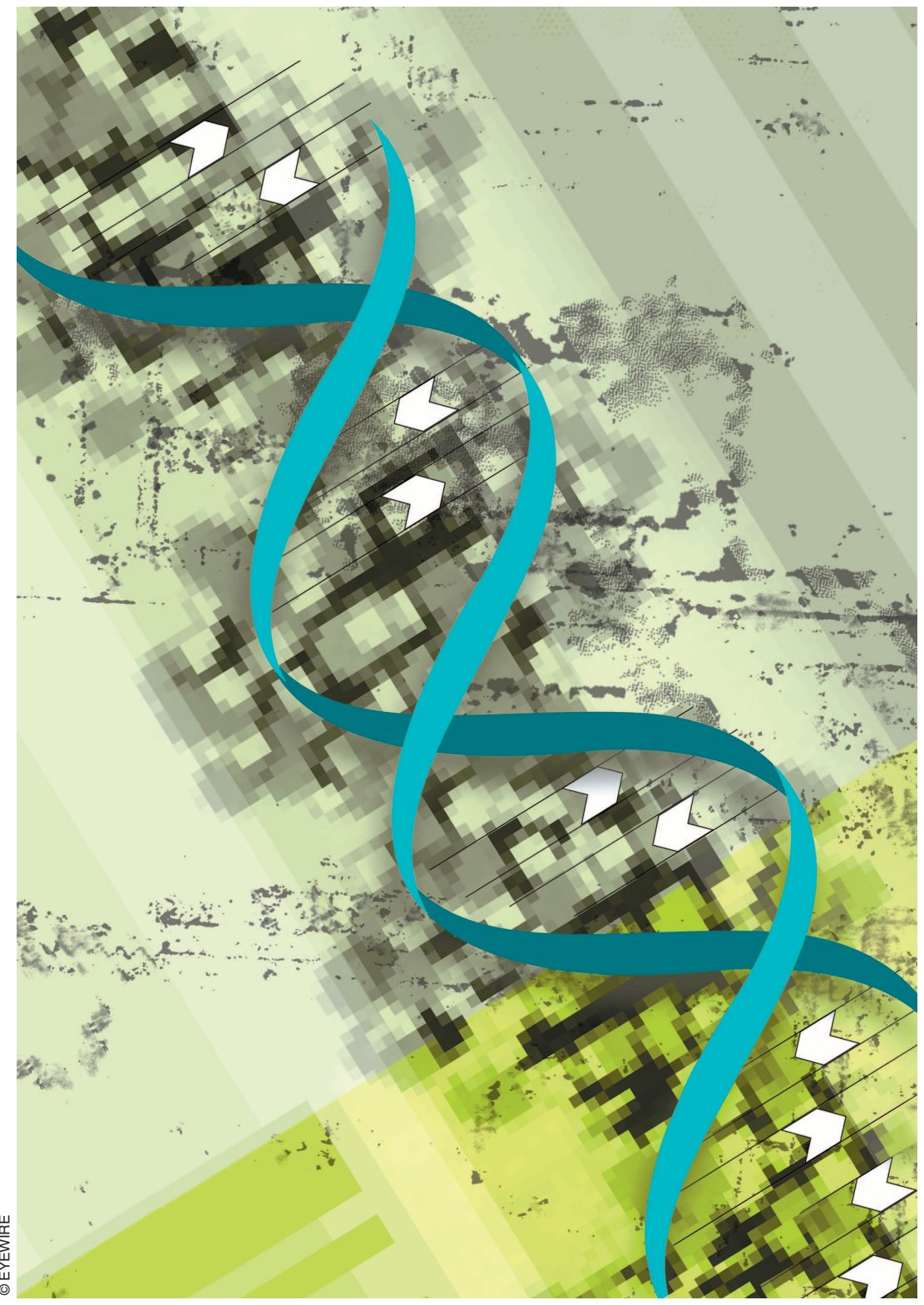

\section{Abstract}

The theory and methods of signal processing are becoming increasingly important in molecular biology. Digital filtering techniques, transform domain methods, and Markov models have played important roles in gene identification, biological sequence analysis, and alignment. This paper contains a brief review of molecular biology, followed by a review of the applications of signal processing theory. This includes the problem of gene finding using digital filtering, and the use of transform domain methods in the study of protein binding spots. The relatively new topic of noncoding genes, and the associated problem of identifying ncRNA buried in DNA sequences are also described. This includes a discussion of hidden Markov models and context free grammars. Several new directions in genomic signal processing are briefly outlined in the end.

Keywords-Genomic-signal-processing, bioinformatics, genes, proteincoding, DNA, and ncRNA. 


\section{Introduction}

$\mathrm{S}$ ubsequent to the sensational announcement of the double helix structure for the DNA molecule more than fifty years ago by Watson and Crick [1], there has been phenomenal progress in genomics in the last five decades. With the enormous amount of genomic and proteomic data available to us in the public domain, it is becoming increasingly important to be able to process this information in ways that are useful to humankind. Traditional as well as modern signal processing methods have played an important role in these fields. Genomic signal processing is primarily the processing of DNA sequences, RNA sequences, and proteins. A DNA sequence is made from an alphabet of four elements, namely $A, T, C$, and $G$. For example

$$
\text { ... ATCCC AAGTATAAGAAGTA... }
$$

The letters $A, T, C, G$ represent molecules called nuclotides or bases (to be described soon). Since DNA contains the genetic information of living organisms, we see that life is governed by quarternary codes. Another example of discrete-alphabet sequences in life forms is the protein. A large number of functions in living organisms are governed by proteins. A protein can be regarded as a sequence of amino acids. There are twenty distinct amino acids, and so a protein can be regarded as a sequence defined on an alphabet of size twenty. The twenty letters used to denote the amino acids are the letters from the English alphabet except $B, J, O, U, X$, and $Z$. For example a part of the protein sequence could be

$$
\text { ...PPVAC ATDEEDAFGGAYPQ .. }
$$

Notice that some letters representing amino acids are identical to some letters representing bases. For example the $A$ in the DNA is a base called adenine, and the $A$ in the protein is an amino acid called alanine.

If we assign numerical values to the four letters in the DNA sequence, we can perform a number of signal processing operations such as Fourier transformation [26, 3], digital filtering [27], time-frequency plots such as wavelet transformations [17], and Markov modelling [4]. Some of those are quite interesting and in fact have important practical applications. Similarly, once we assign numerical values to the twenty amino acids in protein sequences we can do useful signal processing.

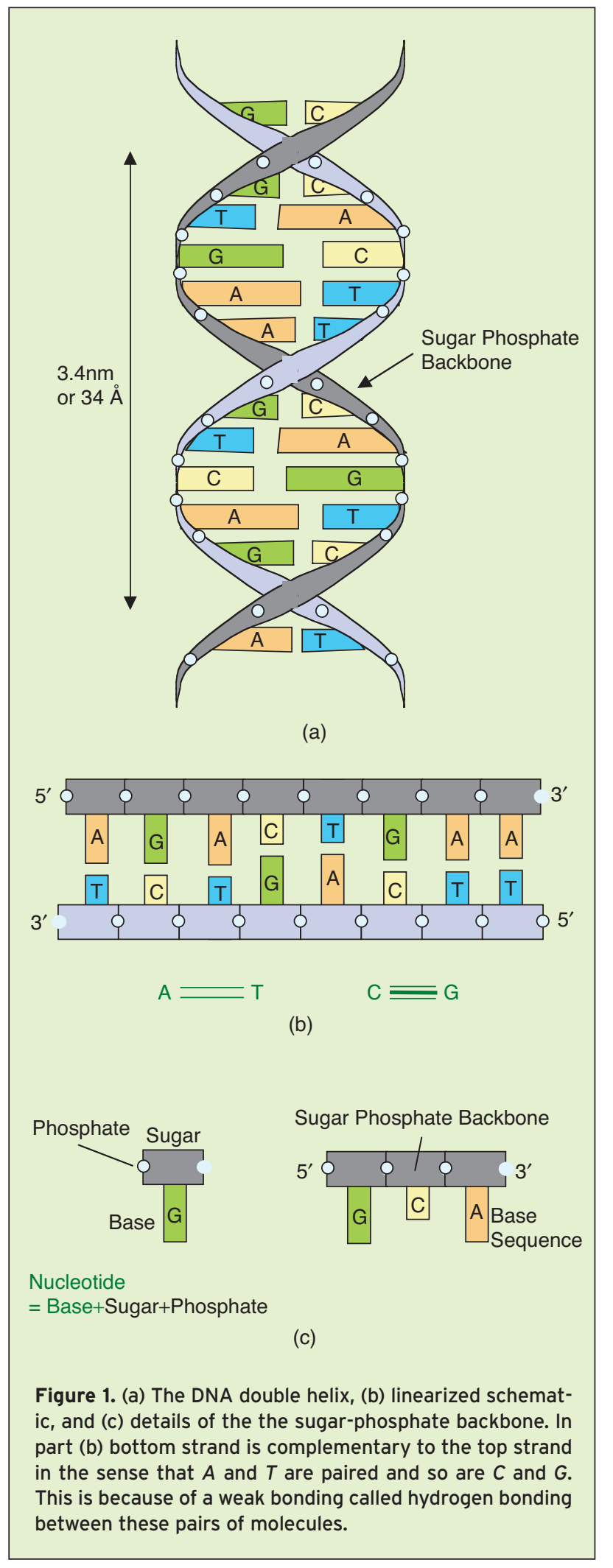

P. P. Vaidyanathan ${ }^{1}$ is with the Department of Electrical Engineering, 136-93, California Institute of Technology, Pasadena, CA 91125. Ph:(626) 3954681 Email: ppvnath@systems.caltech.edu

\footnotetext{
$\overline{{ }^{1} \text { Work supported in part by the ONR grant N00014-99-1-1002. }}$
} 


\section{STORY OF HOW THE DNA DOUBLE HELIX WAS DISCOVERED}

ton he notion that there are specific factors (genes) that are passed on to offspring probably started with the work of Gregor Mendel around 1856. Nearly half a century later it became clear due to the work of Walter Sutton (medical student, Columbia University) and T. H. Morgan (also at Columbia), that these "factors" were located on chromosomes which were known to contain proteins and DNA molecules. In 1930 the DNA was shown to be a long molecule made of the nitrogenous bases A, T, C and $G$.

In those days proteins were considered to be the "genes" that carried hereditary information. In 1944, the experiments of 0 . T. Avery (Rockfeller Inst., NY) showed that DNA, rather than protein, carried genetic traits. For example when a virus attacks bacteria, it is the viral DNA and not the protein that enters the bacteria and changes its behavior. Alfred Hershey and Martha Chase verified this experimentally (1952, Cold Spring Harbor). It was accepted that genes were contained in the DNA; nothing was known about their nature or how they worked.

In 1944, the famous physicist Schrodinger wrote a book entitled What is Life, which inspired many young scientists. J. D. Watson (born in Chicago, 1928) was among them. He was fascinated from childhood by the mystery behind genes. Watson worked on bacteriophages or phages (viruses that attack bacteria) to receive his $\mathrm{Ph}$.D. at the young age of 22 from Indiana University, and later went to the Cavendish Laboratories (Cambridge, England) for further work. The following story is based on his own account of the histo- ry of the double helix [9]. When Maurice Wilkins (King's College, London) showed the X-ray diffraction pattern of DNA to Watson, he got interested in finding the structure of the DNA-that would be the only way to understand genes. Watson worked with Francis Crick at the Cavendish. Earlier, Wilkins had showed the DNA X-ray patterns to a theoretician (Alex Stokes) who said that the pattern must have come from a helix. So Watson and Wilkins were sure it would be a helix. But they thought it would be a triple helix because of the estimated thickness and density known to Wilkins.

Around the same time (1951) Linus Pauling at Caltech (an alltime great chemist) established the $\alpha$-helix structure of the protein molecule. Pauling often worked on macromolecule problems by playing with models which looked like preschool toys (made from balls, sticks, and glue). The success of this method inspired Watson to try a model building approach and hopefully prove that the DNA indeed was a helix. In the meantime, Crick and Bill Cohran (also at Cavendish) developed a theory for the X-ray diffraction patterns from helical structures (the Crick-Cohran-Stokes theory of helical diffraction) and verified that the theory was consistent with Pauling's $\alpha$-helix and its X-ray pattern.

Watson and Crick soon built the triple helix model for DNA. Wilkins and his colleague Rosalind Franklin from King's College, London, visited them and argued that the triple helix was inconsistent with the water content found in DNA (according to X-ray patterns obtained by Franklin). This halted all efforts for a while.

\section{Scope and Outline}

This magazine article is meant only to be an introduction. The aim here is to present a big picture with appropriate background information. The field is quite mature, and the reader with serious interest should pursue some of the references cited at the end of this article. For convenience the references are categorized by topic.

Sections $2-5$ contain brief but important background material on DNA and proteins sequences. In Sec. 6 we explain how Fourier techniques have played a role in gene identification and protein analysis. Sec. 7 explains the role of hidden Markov models in molecular biology. We then discuss in Sec. 8 noncoding genes which have been increasingly recognized for their important role in nearly all life forms. A brief overview of issues involved in computational identification of noncoding genes is also presented. We conclude the paper with further remarks on topics of recent interest. Overviews of some of the important aspects of genomic signal processing can be found in the introductory magazine-article by Anastassiou [3] and in a recent journal article [8].

\section{Some Fundamentals}

Figure 1(a) shows a schematic for the DNA (deoxyribo nucleic acid) molecule. This is in the form of a double helix. The discovery of this double helix is one of the landmarks of molecular biology (for detailed story, see Box B1). Between the two strands of the backbone which is outside, there are pairs of bases like the rungs of a ladder. The backbone is a very regular structure made from sugar-phosphate. There are four types of bases (or nucleotides), denoted with the letters $A, C, G$, and $T$ (respectively, adenine, cytosine, guanine, and thymine). For completeness, the internal atomic details of the molecules $A, T, C$, and $G$ are shown in Figure 2 . These molecules are made from carbon, nitrogen, hydrogen and oxygen atoms. There are about three billion of these bases in the DNA of a single human cell (Figure 3).

In Figure 1(b) the double helix is shown straightened out for simplicity. The genome sequence corresponding to the top strand of the DNA molecule in this example is $A G A C T G A A$. Note that the ordering is from the so-called 
At this time Watson learned that Erwin Chargaff (Columbia University) had shown earlier that the concentration of the bases $A$ and $T$ were the same in DNA samples. So were those of $C$ and $G$. Crick was slowly learning that $A$ and $T$ might stick by hydrogen bonding at their flat surface and so might $C$ and $G$. There were papers by Gulland and Jordan showing that there were lots of hydrogen bondings even at low DNA concentrations. By combining this with Chargaff's observation Crick realized that the DNA molecule might have the bases paired up this way.

Pauling also got interested in finding the DNA structure, and he too came up with a triple helix model! Watson quickly found flaws in the chemistry of the structure: it would make the DNA neutral rather than weakly acidic (as it had earlier been shown to be). Watson shared this message with Wilkins and Franklin during a visit. Wilkins also showed Watson the most recent X-ray pictures of DNA taken by Franklin and her student Gosling. These were great pictures of the $B$-form DNA taken with some meticulous effort, and it immediately became obvious to Watson that the molecule ought to have a helical structure. (They were studying two forms of DNA, the crystalline (A form) and paracrystalline (B form).) Later he could even deduce that it implied $3.4 \mathrm{~nm}$ periodicity (Fig. 1).

Then Watson and Crick decided to build again models for the DNA helix. This time they tried the double helix model first, the joke being that all biological objects came in pairs [9].
From the 1951 work of Alexander Todd (Cambridge, England) they knew that the backbone of the DNA molecule was very regular (today known to be the sugar-phosphate backbone). Watson and Crick first tried a model where like-bases stuck together ( $A$ with $A, T$ with $T$, and so on) by hydrogen bonding. This wrong path was chosen because they were using a wrong chemical configuration for the bases called the enol form. The American crystallographer Jerry Donohue at Cavendish convinced them to use the so-called keto form in the models. When attempting this, Watson made the most crucial discovery that the base $A$ in one strand had to pair with $T$ in the other. Similarly $C$ and $G$ would have to pair. Such pairs are held together by hydrogen bonding, and furthermore have similar shape. The resulting double helix was verified to be correct stereochemically, in addition to being consistent with X-ray diffraction patterns. It was also consistent with Chargaff's earlier observation that some bases have identical concentrations in DNA. The resulting model was readily accepted by Wilkins, Franklin, and Pauling. "A structure as pretty as that just had to be right!"

Watson and Crick had won the race. Their paper announcing the double helix appeared in the journal Nature on April 25, 1953a one-page paper reporting one of the greatest discoveries of science! In 1962 when Watson was 34, he shared the Nobel prize for Physiology or Medicine with Crick and Wilkins. $5^{\prime}$ to the $3^{\prime}$ end (left to right). DNA sequences are typically listed from the $5^{\prime}$ to the $3^{\prime}$ end because they are scanned in that direction when bases are used by the cell machinery to signal the production of amino acids. The reason for directed flow arises from the way the sugar and phosphate are glued together (Figure 1(c)). In the double stranded DNA, the base $A$ always pairs with $T$, and $C$ pairs with $G$. Thus the bottom strand TCTGACTT is the complement of the top strand. This is called the Watson-Crick base-pairing; it occurs through a weak bond called the hydrogen bond [2] but because there are several million base pairs, the two strands are held together strongly. Typically in any given region of the DNA molecule, at most one of the two strands is active in gene expression (Sec. 3).

Single-celled organisms like bacteria do not have a nucleus and the DNA just resides in the cell. Such cells are called procaryotes; higher organisms (worms, insects, plants, mammals, ...) have cells with nucleus and are called eucaryotes. These have the DNA residing in the nucleus. An exception is the red blood cell which has no nucleus. Cells also have a small quantity of DNA in the mitochondria; we shall not discuss this here.

The RNA (ribo nucleic acid) molecule is closely related to the DNA. It is also made of four bases but instead of thymine, a molecule called uracil is used (denoted as $U$ ). The sugar in the sugar-phosphate backbone is also slightly different but we do not require the details here. The important fact is that $U$ pairs with $A$ by hydrogen bonding just like $T$ pairs with $A$. RNA molecules are short (and typically short-lived) single-stranded molecules which are used by the cell as temporary copies of portions of DNA (Sec. 3).

\section{Genes and DNA}

A DNA sequence can be separated into two types of regions: genes and intergenic spaces. Genes contain the information for generation of proteins. Each gene is responsible for the production of a different protein as shown schematically in Figure 4. Even though all the cells in an organism have identical genes, only a selected subset is active in any particular family of cells. For example 


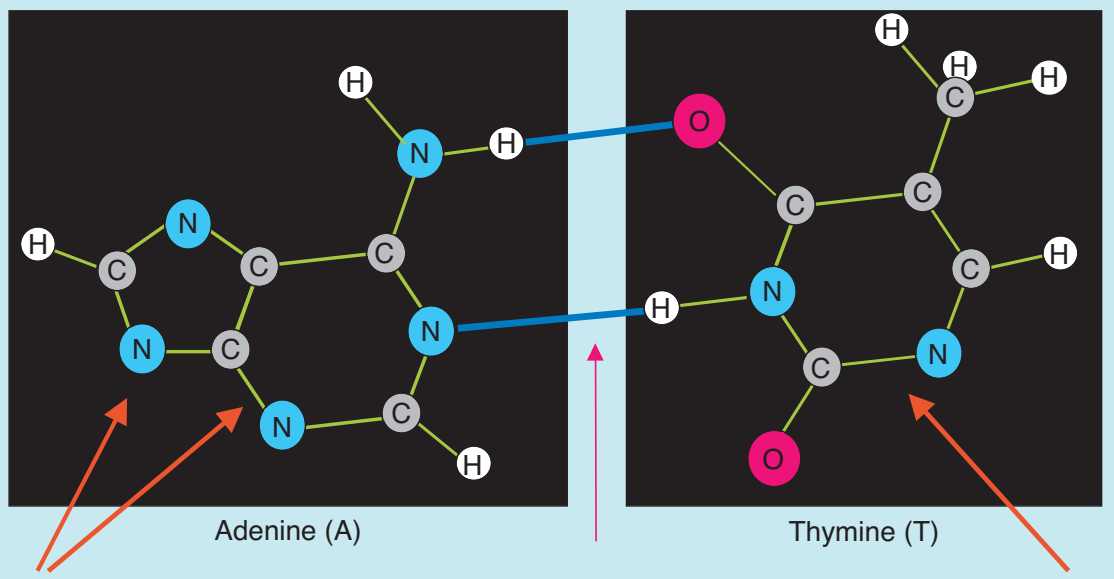

Purine (2 Rings)

$$
\begin{aligned}
& \mathrm{A}=\mathrm{T} \text { Bonding } \\
& \mathrm{A}=\mathrm{U} \text { is Similar }
\end{aligned}
$$

Double Hydrogen-Bond

(a)

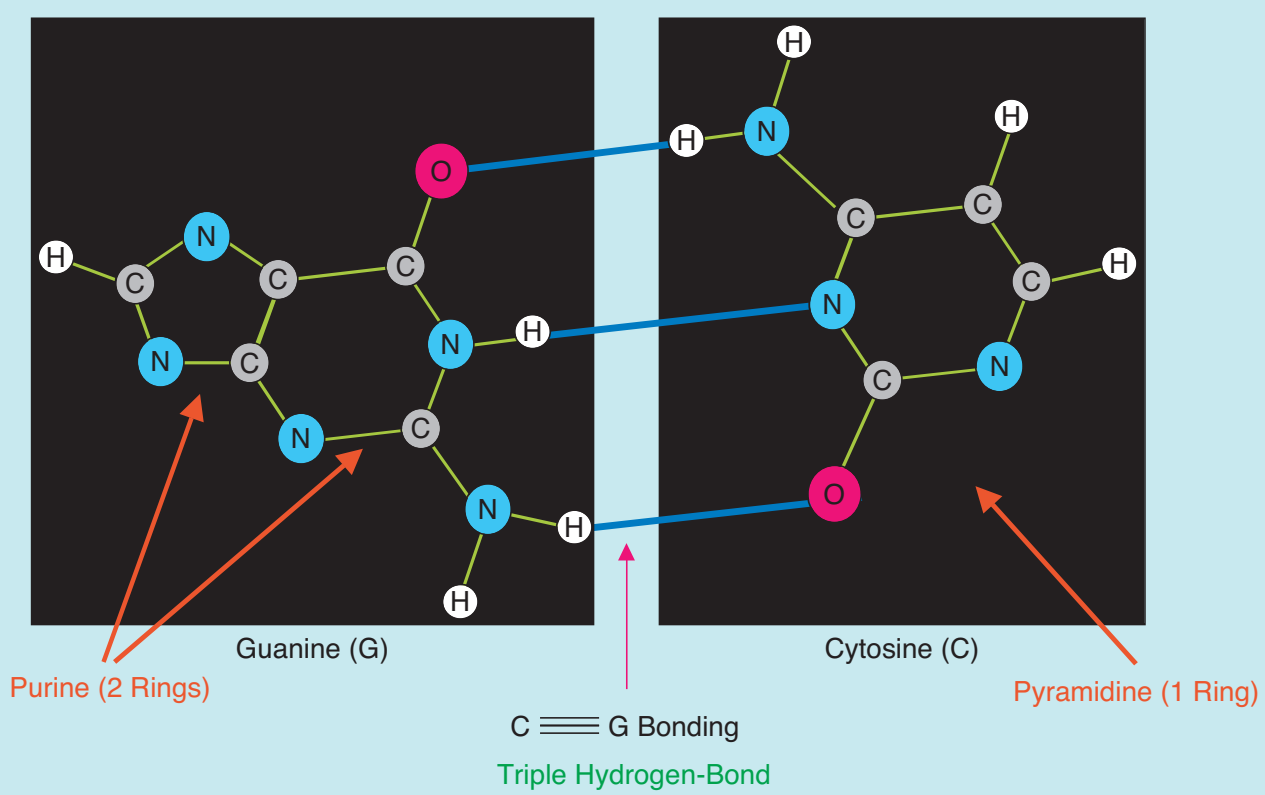

(b)

Figure 2. Internal atomic details of the bases adenine and thymine (a), guanine and cytosine (b). These molecules are made from carbon, hydrogen, oxygen and nitrogen (hence called nitrogenous bases). Note that $A$ and $G$ have two rings and are called purines. The molecules $C$ and $T$ have one ring and are called pyramidines.

the set of genes that are active in blood cells are different from those that are active in nerve cells, which explains why these cells look so different! See Figure 5.

Figure 6 shows some of the steps involved in the production of a protein from a gene. Notice that a gene has two types of subregions called the exons and introns (procaryotes like bacteria do not have introns). ${ }^{2}$ The gene is first copied into a single stranded chain called the messenger RNA or mRNA molecule. The introns are then removed from the mRNA by a process called splicing. The spliced mRNA is then used by a large molecule called the ribosome to produced the appropriate protein. The translation from mRNA to protein is aided by adaptor molecules called the transfer RNA or tRNA molecules. In some sense the tRNA molecules store the genetic code as

2 The existence of introns came to the attention of the scientific community only in 1977 [2]. 
we shall see in Sec. 4. Ribosomes are often referred to as the protein factories of the cell. There are many ribosomes in a cell working in parallel like molecular machines.

Many details are omitted in Figure 6 for brevity. For example the mRNA is in reality the complement of the gene, that is, $C$ s are replaced with $G$ s, and $A s$ with $T s$ (rather Us). Thus, if the gene is ATTAGC then the mRNA is $U A A U C G$. There is a second level of complementing which cancels this when the mRNA attaches to tRNA molecules at the so-called anticodon sites.

The observation that each gene is responsible for the creation of a protein (through mRNA) is often expressed as

gene in $D N A \rightarrow R N A \rightarrow$ protein

and is referred to as the central dogma of molecular biology. We will see in Sec. 8. that the dogma has been challenged in recent years.

\section{The Genetic Code}

How does the cell know what protein to make from a particular gene? This information is contained in a code which is common to all life. Recall that the gene gets duplicated into the mRNA molecule which is then spliced so that it contains only the exons of the gene. Imagine that this spliced mRNA is divided into groups of three adjacent bases. Each triplet is called a codon. Evidently there are 64 possible codons. Thus the
mRNA is nothing but a sequence of codons. Each codon instructs the cell machinery to synthesize an amino acid. The codon sequence therefore uniquely identifies an amino acid sequence which defines a protein. This mapping is called the genetic code and is shown ${ }^{3}$ in Figure 7. Since there are 64 possible codons but only 20 amino acids, the mapping from codons to amino acids is many-

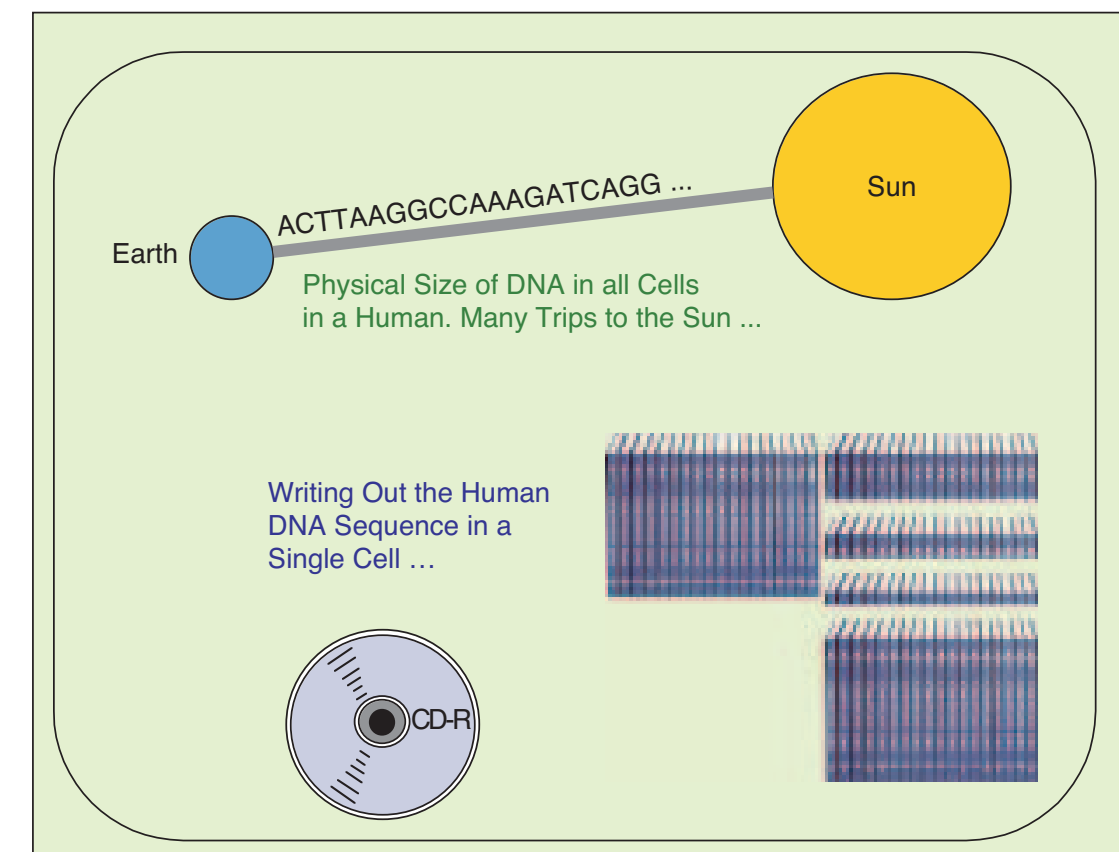

Figure 3. A feeling for sizes ... The DNA in the nucleus of a single human cell is about 3 billion bases long (and is organized into 46 chromosomes). For typical bacteria the DNA is about 4 million bases long. If the DNA in a human cell is stretched out like a piece of string, it stretches out to 2 yards! If we put together all DNA in all the ( 5 trillion) cells in an average human, the length is sufficient to cover the distance from earth to the Sun (93 million miles), about 50 times. If we were to write down each base using normal letter size, the DNA in a single human cell would fill about 2000 novels. If the three billion bases in a human genome are stored digitally using two bits to code each base location (of four possible bases), the total is 6 billion bits or equivalently 750 Mega bytes (roughly the capacity of a standard CD). A typical cell nucleus which is one hundredth of a millimeter across can store as much information as a does CD!

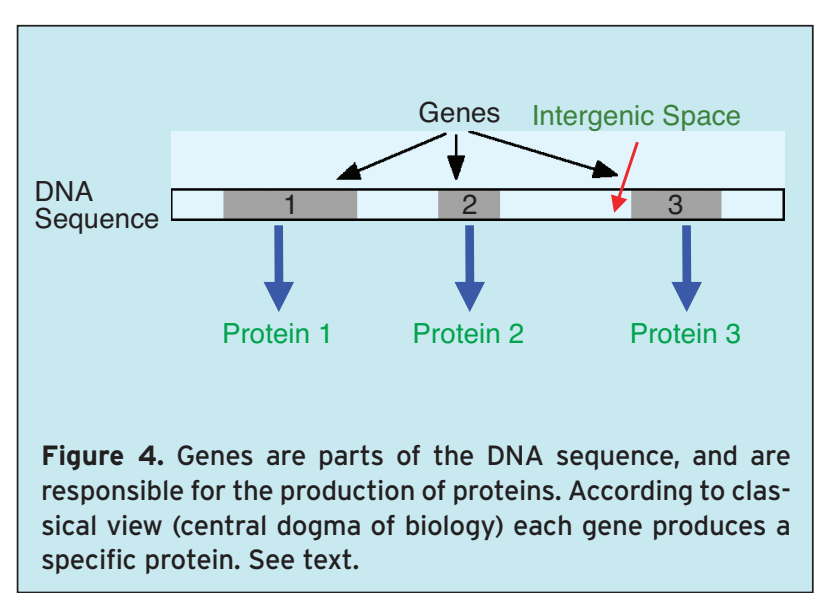

FOURTH QUARTER 2004
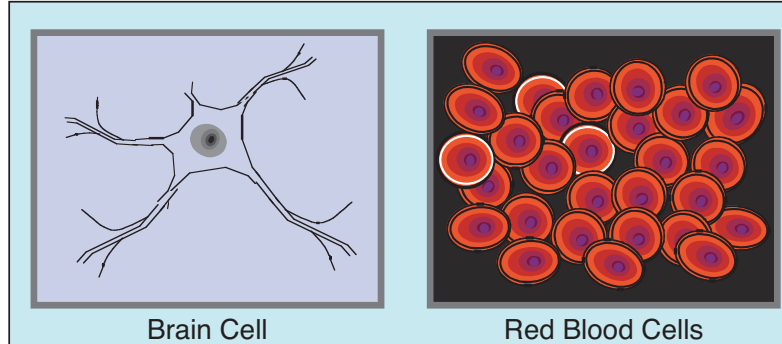

Figure 5. Brain cells and red blood cells. Cells look very different from each other because of the different sets of genes expressed in them. See www-biology.ucsd.edu/ news/article_112901.html and www.cellsalive.com/gallery. htm for real micrographs. 


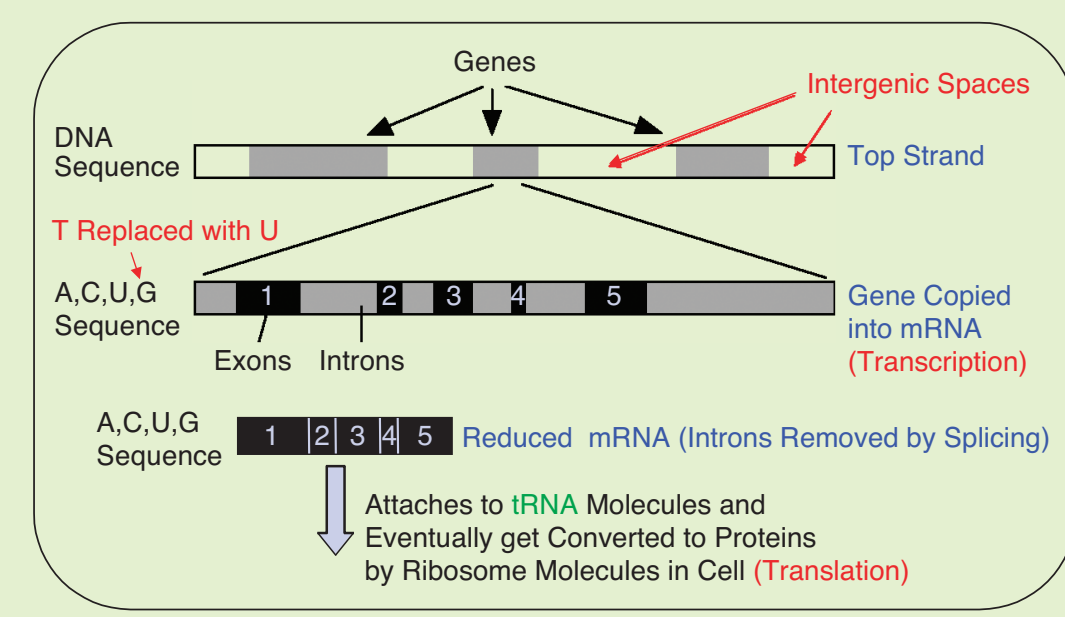

Figure 6. When a gene is ready to be expressed, it is duplicated in the form of a singlestrand molecule called the mRNA (messenger RNA) which then leaves the nucleus. The introns are spliced out and a shorter mRNA molecule is produced. Thus, unlike the parent gene, the mRNA is a concatenation of the exons only. It is used by ribosomes outside the nucleus of the cell to manufacture the appropriate protein coded by the original gene. Thus protein production involves the transcription of genes into mRNA and the subsequent translation of the 4-letter language to a 20-letter language.

\begin{tabular}{|c|c|c|c|}
\hline AAA: K (Lys) & GAA: $\mathrm{E}$ (Glu) & TAA: Stop & $C A A: Q(G \mid n)$ \\
\hline AAG: K (Lys) & GAG: E (Glu) & TAG: Stop & CAG: $Q$ (GIn) \\
\hline AAT: N (Asn) & GAT: D (Asp) & TAT: Y (Tyr) & CAT: H (His) \\
\hline$A A C: \mathrm{N}$ (Asn) & GAC: D (Asp) & TAC: Y (Tyr) & CAC: $\mathrm{H}$ (His) \\
\hline AGA: $\mathrm{R}$ (Arg) & GGA: G (Gly) & TGA: Stop & CGA: R (Arg) \\
\hline AGG: $\mathrm{R}$ (Arg) & GGG: G (Gly) & TGG: W (Trp) & CGG: R (Arg) \\
\hline AGT: S (Ser) & GGT: G (Gly) & TGT: C (Cys) & CGT: R (Arg) \\
\hline AGC: S (Ser) & GGC: G (Gly) & TGC: C (Cys) & CGC: R (Arg) \\
\hline ATA: I (Ile) & GTA: V (Val) & TTA: L (Leu) & CTA: L (Leu) \\
\hline ATG: M (Met) & GTG: V (Val) & TTG: L (Leu) & CTG: L (Leu) \\
\hline$A T G=$ Start & & & \\
\hline ATT: I (lle) & GTT: V (Val) & TTT: $\mathrm{F}$ (Phe) & CTT: L (Leu) \\
\hline ATC: I (Ile) & GTC: V (Val) & TTC: F (Phe) & CTC: L (Leu) \\
\hline ACA: $\mathrm{T}$ (Thr) & GCA: A (Ala) & TCA: S (Ser) & CCA: P (Pro) \\
\hline ACG: T (Thr) & GCG: A (Ala) & TCG: S (Ser) & CCG: P (Pro) \\
\hline ACT: T (Thr) & GCT: A (Ala) & TCT: $\mathrm{S}$ (ser) & CCT: P (Pro) \\
\hline ACC: T (Thr) & GCC: A (Ala) & TCC: S (Ser) & CCC: P (Pro) \\
\hline
\end{tabular}

Figure 7. The genetic code. Triples of bases such as AAA denote codons. The single letters such as $\mathrm{K}$ denote amino acids. Their three letter names (e.g., Lys) are also shown. Full names of amino acids can be found in Figure 8.

to-one (Figure 8). The story of how the genetic code was cracked is summarized in Box B2.

When a gene is expressed, each codon in the mRNA produces an amino acid according to the genetic code,

\footnotetext{
${ }^{3}$ We have used $\mathrm{T}$ instead of $\mathrm{U}$ because the original gene has $\mathrm{T}$. In fact, we will use $\mathrm{U}$ and $\mathrm{T}$ rather interchangeably; the context will make the distinction clear.
}

and the amino acids are bonded together into a chain. Figure 9 shows an example of how mRNA is converted to protein using the genetic code. When all the codons in the mRNA are exhausted we get a long chain of amino acids (typically a few hundred long). This is the protein corresponding to the original gene. Notice that there is a start codon ATG which signifies the beginning of the protein-coding part of the gene. If a start codon occurs inside a gene again, it produces the amino acid methionine. A stop codon signifies that the protein coding part of the gene has come to an end. There are three stop codons. The chemical bond between amino acids is a covalent peptide bond. Figure 10 shows examples of two amino acids and the resulting bond.

The translation of the codons into amino acids is made physically possible by adaptor molecules called transfer RNA or tRNA molecules. There are more than 20 kinds of tRNA molecules in the cell (at least one for each amino acid). One end of the molecule matches a specific codon and the other end attaches to the corresponding amino acid. See Figure 11. The molecule ribosome (Sec. 3) works in conjunction with tRNA molecules and mRNA to produce the protein. So it is clear that the genetic code is essentially stored in the tRNA molecules.

It is a wonder of Nature that all life forms (from bacteria to mammals) use the same genetic code. This is no doubt due to the common origin of all life. Can one change Nature's genetic code? Apparently this is not impossible. Recall from Figure 7 that the stop codon TAG produces no amino acid. In 2001 Wang and Schultz added enough biological machinery in E. coli bacteria to enable it to synthesize a new amino acid from TAG. In 2003 they showed how this amino acid can be inserted in a E. coli protein made with natural amino acids. The same idea was successful in 
yeast. It has been suggested by some authors that such new proteins could be the key to destroying cancerous cells quickly. A Scientific American article which appeared in May 2004 describes some of these areas of research [44].

\section{Proteins}

Because of the innumerable combinations from the alphabet of 20 amino acids, the number of different proteins in living organisms is enormous. Proteins drive most of the biological processes in living organisms. Enzymes, for example, are proteins with a special role, namely the speeding up of biochemical reactions in living organisms. Fibers in tendons and ligaments, components of hemoglobin (oxygen carrier in red blood cells), myosin in muscle cells (motor protein), ferritin in the liver, rhodopsin in retina (light detector) and hormones such as insulin, gastrin, and glucagon, are all proteins. When a protein is left in a watery medium it automatically folds into a specific three dimensional structure, which depends almost entirely on the amino acid sequence defining the protein (the $\mathrm{pH}$ or acidity of the watery medium is also important). The 3D shape of a protein allows it to interact only with very specific molecules in the cell, and this is important for the proper functioning of proteins. ${ }^{4}$ In fact protein folding is a major area of research by itself. For example, given the amino acid sequence alone, can we predict the 3D folded shape using physics and mathematics alone? Figure 12 shows a computer drawing of the protein hemoglobin which is made of four smaller proteins [2]. Like DNA, proteins are macro molecules. The average protein is about 40,000 times heavier than a hydrogen atom. We will say more about the signal processing aspects in Sec. 6.3.

The discovery of the double

\begin{tabular}{|c|c|c|c|c|}
\hline 1 & $A$ & Ala & Alanine & GCA, GCC, GCG, GCT \\
\hline 2 & C & Cys & Cysteine (has S) & TGC, TGT \\
\hline 3 & $D$ & Asp & Aspartic acid & GAC, GAT \\
\hline 4 & $E$ & Glu & Glutamic acid & GAA, GAG \\
\hline 5 & $F$ & Phe & Phenylalanine $^{1}$ & TTC, TTT \\
\hline 6 & $G$ & Gly & Glycine & GGA, GGC, GGG, GGT \\
\hline 7 & $H$ & His & Histidine $^{2}$ & CAC, CAT \\
\hline 8 & $I$ & lle & Isoleucine ${ }^{3}$ & ATA, ATC, ATT \\
\hline 9 & $K$ & Lys & Lysine $^{4}$ & AAA, AAG \\
\hline 10 & $L$ & Leu & Leucine $^{5}$ & TTA, TTG, CTA, CTC, CTG, CTT \\
\hline 11 & $M$ & Met & Methionine $^{6}$ (has S) & ATG \\
\hline 12 & $N$ & Asn & Asparagine & AAC, AAT \\
\hline 13 & $P$ & Pro & Proline & CCA, CCC, CCG, CCT \\
\hline 14 & $Q$ & Gln & Glutamine & CAA, CAG \\
\hline 15 & $R$ & Arg & Arginine $^{7}$ & AGA, AGG, CGA, CGC, CGG, CGT \\
\hline 16 & $S$ & Ser & Serine & AGC, AGT, TCA, TCC, TCG, TCT \\
\hline 17 & $T$ & Thr & Threonine $^{8}$ & ACA, ACC, ACG, ACT \\
\hline 18 & $V$ & Val & Valine $^{9}$ & GTA, GTC, GTG, GTT \\
\hline 19 & $W$ & Trp & Tryptophan ${ }^{10}$ & TGG \\
\hline 20 & $Y$ & Tyr & Tyrosine $^{11}$ & TAC, TAT \\
\hline
\end{tabular}

Figure 8. A list of the twenty amino acids, and codons which generate them (from Fig. 7). For example the amino acid alanine $(A)$ can be generated by any one of four possible codons GCA, GCC, GCG, or GCT. The superscipts 1 to 11 indicate the eleven essential amino acids (some references say there are fewer than eleven). These by definition are the amino acids animals cannot manufacture-they need to eat them. Milk provides all essential amino acids, and so does a combination of grains and beans.

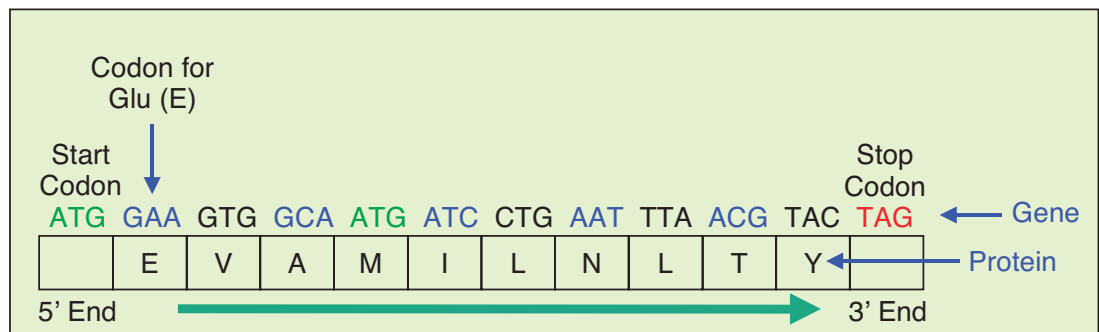

Figure 9. A toy example showing how a sequence of codons gets translated to a protein, ten amino-acids long. In most cases genes are much longer (thousands of bases); proteins have several hundred amino acids. Notice that if a base is deleted by accident somewhere in the middle, then all the codons following that point are changed, possibly changing all the amino acids that follow. If an entire codon is deleted, it is like deleting an amino acid; nothing else changes.

helix also solved another mystery of molecular biology: it suggested how the huge DNA is replicated accurately

\footnotetext{
${ }^{4}$ For example, the enzyme thrombin reacts only with the protein fibrinogen (which is a part of the blood clotting process). There are exceptions too: the digestive enzymes pepsin and chymotrypsin act on almost any protein they encounter. The Encyclopedia Britannica contains a wealth of information on this topic.
}

in cell division. Namely, the double strand separates or unzips into two single strands each of which serves as a mold to form a new complementary strand. (The unzipping process is also present locally when a gene is copied into an mRNA (Figure 13)). Each single strand quickly manufactures the complementary strand from bases floating around in the cell. This was later verified 


\section{STORY OF HOW NATURE'S GREATEST CODING MYSTERY WAS CRACKED}

erhaps the earliest proposal that genes did their work by generating proteins came in 1941 from Beadle and Tatum at Stanford. They worked with mold which grew on bread and argued that X-rays create changes (mutations) in some genes, affecting the generation of certain proteins (enzymes, to be specific). About ten years later, Linus Pauling and Harvey Itano at Caltech had evidence, based on their work on hemoglobin proteins, that each protein might have an associated gene. They showed sickle cells were caused by one single change in the amino acid chain (see Fig. 14). Then the famous physicist George Gamow proposed many possible mappings from DNA to protein, but nothing worked for a while.

The prediction that there ought to be an intermediate RNA molecule between DNA and protein was made first by Watson even before the double helix was invented. From this arose the central dogma of biology (Sec. 3) which is often credited to Crick who did much to popularize it. In a 1955 private communication to the RNA tie club members (a club founded by George Gamow [10]) Crick suggested that there ought to be an adaptor molecule for every amino acid, later found to be the tRNA. But the way in which it turns DNA into protein was not clear. In 1959 an enzyme called the RNA polymerase was discovered. It was involved in the production of single stranded RNA from double stranded DNA. The great moment came when the ribosome was discovered at the Massachusetts General Hospital, Boston. Here Paul Zamecnik was studying cell-free protein synthesis and could track amino acids radioactively. He found that they were being strung together at the sites of small molecules in the cell today known as the ribosomes. Zamecnik then worked with Mahlon Hoagland and showed that before these amino acids were assembled into a chain at the ribosome they were attached to some small RNA molecules. Watson and Crick pointed out that these ought to be the adaptors they were looking for, today known as the transfer RNA or tRNA molecules. The messenger RNAs (mRNAs) were verified to be the templates for proteins synthesis only in 1960. Details of the complete story (starting from the DNA through mRNA to protein) was worked out at Harvard, Caltech, and Cambridge (Watson, Matt Meselson, Francois Jacob, and Sydney Brenner).

The code that translates portions of DNA into specific sequences of amino acids came up next. Since there are only four choices for bases in DNA, a single base is not enough to specify one out of 20 amino acids. A sequence of three consecutive bases has $4^{3}=64$ combinations, so Sydney Brenner proposed that the transcription from the 4-letter DNA to the 20-letter protein takes place through triplets of bases (now called codons), each triplet specifying one amino acid. In 1961 Brenner and Crick at the Cambridge Labs then proved this experimentally, by deleting or inserting base pairs in DNA and seeing the effect on the resulting amino acid sequences. This was the first experimental proof of the existence of codons. The ability to force artificial mutations (insertion, deletion and alteration of bases) was crucial to these experiments. Also crucial was the fact that protein synthesis could be performed outside the cell in a test tube using a good supply of ribosomes, amino acids, transfer RNAs and a source of energy. Such a system would manufacture the proteins that correspond to an mRNA introduced into the test tube.

In 1961 Marshall Nirenberg from the National Institute of Health revealed at a conference in Moscow that the triplet TTT produces the amino acid phenylalanine (Phe or F). He found this by using the RNA molecule UUUUUU ... (called poly-U) in a cell-free synthesis of amino acids. Thus $1 / 64$ th of the genetic code had been cracked. Still there remained 63 triplets of bases for which the resulting amino acids had to be found out. This was completed in 1966 by Gobind Khorana at U. Wisconsin, and the complete genetic code had been cracked! The results were presented at the 1966 Symposium on genetic code in Cold Spring Harbor. The Nobel prize for this work went to Khorana, Nirenberg and Holley in 1968.

by Matt Meselson and Frank Stahl, sometime after 1954, in an experiment considered to be one of the most beautiful experiments in biology. The accuracy of duplication is phenomenal because of the self error correcting mechanism (called mismatch-pair system) implicit in the cell [2]. The probability of error is about $10^{-9}$. Compare this to a average typist (1 error per typed page) or the postal system (10 late deliveries out of every...). Such accuracy is necessary in gene reproduction because even small changes in the DNA (mutations, insertions, deletions) can change the proteins made by the genes dramatically. For example, sickle cell anemia is created because of a single error in a gene (see Figure 14). On the other hand there are examples where even multiple errors do not change the protein (because the codon to amino acid mapping has redundancy, Figure 8. So the cell has built-in tolerance for errors; the example of sickle cell anemia is rather unusual.

\section{Filtering and Transform-Domain Methods in Genomics and Proteomics}

The application of Fourier transform techniques has been found to be very useful both for DNAs and protein sequences. First it is convenient to introduce indicator 


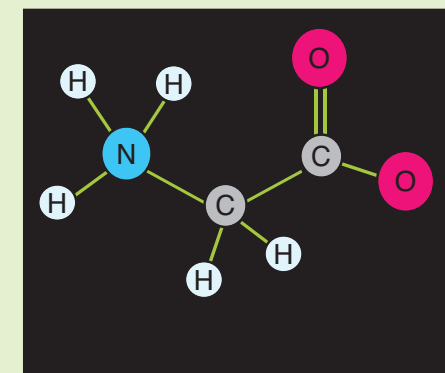

Glycine $(G)$

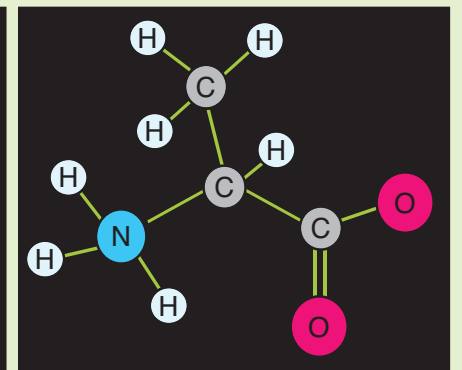

Alanine (A)

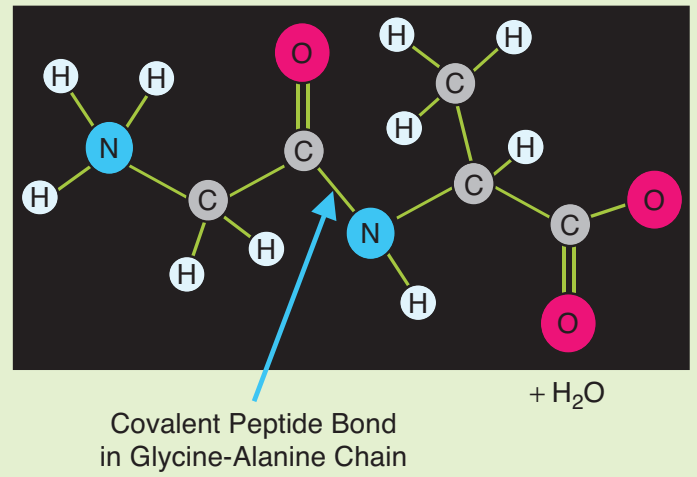

(b)

Figure 10. (a) Examples of two amino acids, and (b) bonding of these two amino acids, with consequent release of a water molecule. Like bases, amino acids are also made from carbon, hydrogen, oxygen, and nitrogen. Some of them also have sulfur (as indicated in Figure 8).

sequences for bases in DNA. For example the indicator for base $A$ is a binary sequence of the form $x_{A}(n)=$ $000110111000101010 \ldots$, where 1 indicates the presence of an $A$ and 0 indicates its absence. The indicator sequences for the other bases are defined similarly. Denote the discrete Fourier transform [64] or DFT of a length- $N$ block of $x_{A}(n)$ as $X_{A}[k]$, that is, $X_{A}[k]=\sum_{n=0}^{N-1} x_{A}(n) e^{-j 2 \pi k n / N}, \quad 0 \leq k \leq N-1$. The DFTs $X_{T}[k], X_{C}[k]$, and $X_{G}[k]$ are defined similarly.

\subsection{Identifying Protein Coding Genes}

It has been noticed that protein-coding regions (exons) in genes have a period-3 component because of coding biases in the translation of codons into amino acids. This observation can be traced back to the 1980 work of Trifonov and Sussman [35]. The period-3 property is not present outside exons, and can be exploited to locate exons. [3, 26]. Thus if we take $N$ to be a multiple of 3 and plot

$$
S[k] \triangleq\left|X_{A}[k]\right|^{2}+\left|X_{T}[k]\right|^{2}+\left|X_{C}[k]\right|^{2}+\left|X_{G}[k]\right|^{2}
$$

then we should see a peak at the sample value $k=N / 3$ (corresponding to $2 \pi / 3$ ). Given a long sequence of bases we can calculate $S[N / 3]$ for short windows of the data, and then slide the window. Thus, we get a picture of how $S[N / 3]$ evolves along the length of the DNA sequence. It is necessary that the window length $N$ be sufficiently large (typical window sizes are a few hundreds, e.g., 351, to a few thousands) so that the periodicity effect dominates the background $1 / f$ spectrum (Sec. 6.2). However, a long window implies longer computation time, and also compromises the base-domain resolution in predicting the exon location.

The sliding window method can be regarded as digital filtering followed by downsampling (at a rate depending on the separation between adjacent positions of the win- dow [67]). The filter has a simple impulse response

$$
w(n)= \begin{cases}e^{j \omega_{0} n} & 0 \leq n \leq N-1 \\ 0 & \text { otherwise }\end{cases}
$$

This is a bandpass filter with passband centered at

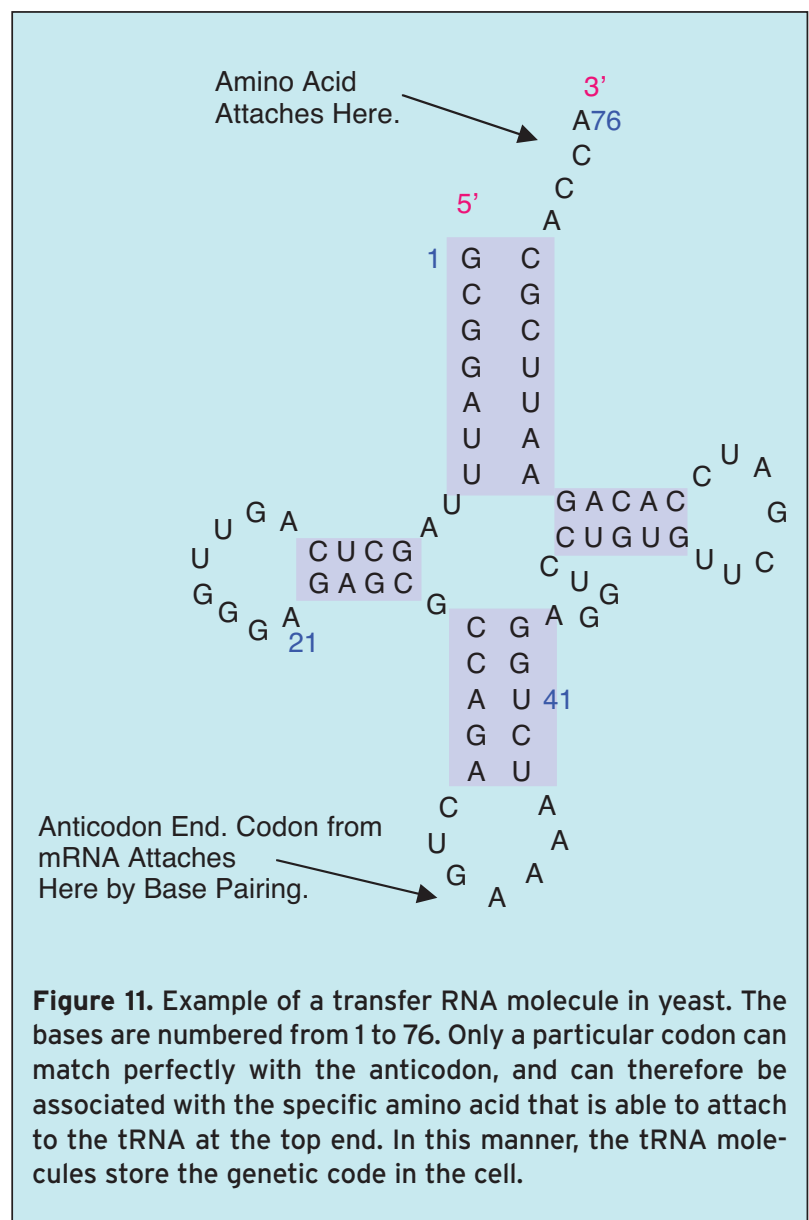




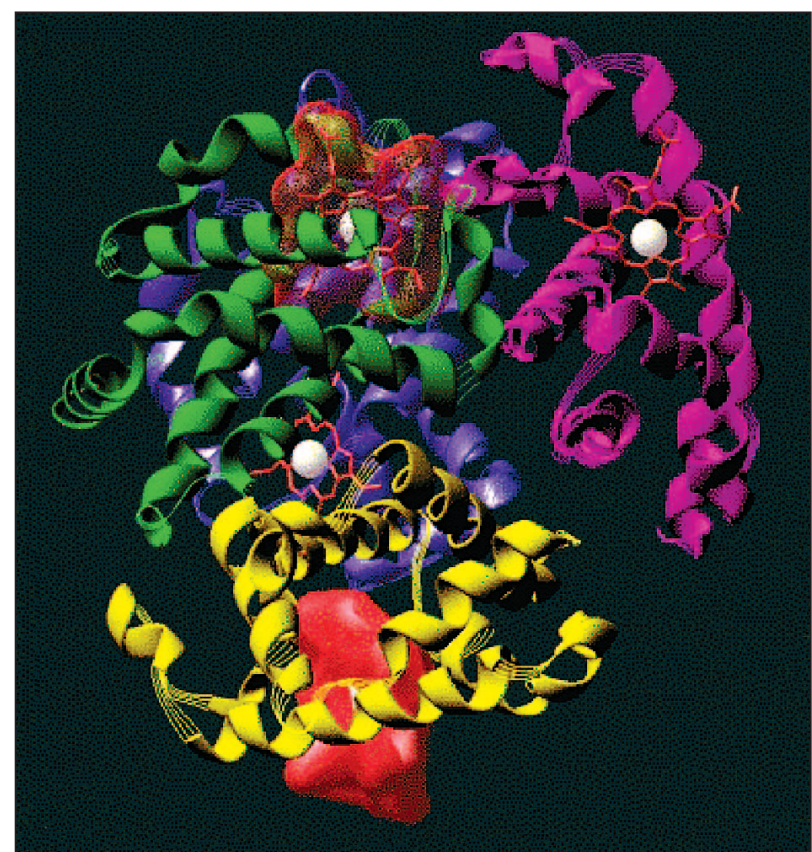

Figure 12. Pasta dish? No, it is an example of a protein (Hemoglobin, human). Figure taken from the website www.biochem.szote.u-szeged.hu/astrojan/protein2.htm, generated by the program MOLMOL (Koradi et al., 1996). See reference [15].

$\omega_{0}=2 \pi / 3$ and minimum stopband attenuation of about $13 \mathrm{~dB}$ (Figure 15). If we pay careful attention to the design of the digital filter, we can isolate the period-3 behavior from background information such as $1 / f$ noise more effectively. We can also use efficient methods to design and implement the filter, thereby reducing computational complexity.

Based on these observations, a number of methods have been proposed for designing digital filters suited to gene prediction application [27], [28]. We show in Figure 16 the exon prediction results for gene F56F11.4 in the C. elegans chromosome III. This gene has five exons. The first plot uses the DFT based spectrum using a sliding window. The five peaks corresponding to the exons can be seen clearly. The second plot uses a multistage filter $H(z)$ similar to the IFIR filter advanced by Neuvo et al. [63]. Notice that the background noise (due to $1 / f$ behavior, Sec. 6.2) has been removed almost completely and the five exons can be seen clearly. Further design details can be found in [28] and in a recent tutorial article [8].

Some authors have claimed that the period-3 property is due to nonuniform codon usage, also known as codon bias; even though there are several codons which code a given amino acid (Figure 8), they are not used with uniform probability in organisms. For example, base $G$ dominates at certain codon positions in the coding regions [23]. We have, in fact, observed experimentally that the use of the plot $\left|X_{G}(k)\right|^{2}$, which depends on base $G$ alone, is often quite sufficient for revealing the period-3 property, and therefore for the prediction of protein coding regions.

Does the method always work? Tiwari et al. [26] have observed that some genes do not exhibit period-3 behavior at all in S. cerevisiae. Furthermore, for procaryotes (cells without a nucleus), and some viral and mitochondrial base sequences, such periodicity has even been observed in noncoding regions [33]. For this and many other reasons [23], gene identification is a very complex problem, and the identification of period-3 regions is only a step towards gene and exon identification. Hidden Markov models (Sec. 7) have been used quite successfully for this application [24].

\subsection{Long Range Correlations or 1/f Behavior}

The period-3 behavior described above indicates a strong short-term correlation in the coding regions. But there is also a long-range correlation exhibited by DNA sequences both in the gene regions and intergenetic regions. One of the earliest papers to point this out appeared in Nature in 1992 [34]. The study made was based on a concept called the DNA walk. Latter studies by other authors examined correlations over much longer regions which contained

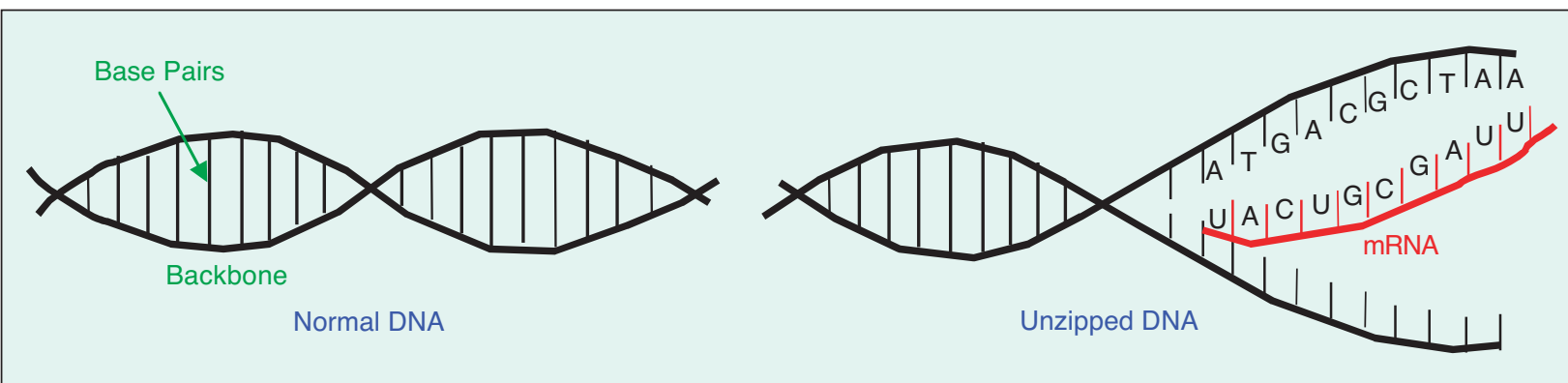

Figure 13. Unzipping of a DNA sequence to produce an mRNA copy of a selected region. This occurs during gene expression. Note that the mRNA strand is complementary to the DNA, that is $A$ is replaced with a $T$ (rather $U$ which is similar) and vice versa; similarly $C$ is replaced with $G$ and vice versa. A similar unzipping separates the two DNA strands completely during cell division. 


\section{CTG ACT CCT GAG GAG AAG TCT Normal Gene leu thr pro glu glu lys ser \\ CTG ACT CCT GTG GAG AAG TCT Mutant Gene leu thr pro val glu lys ser}

Figure 14. Cause of sickle-cell anemia. A gene called HBB in human chromosome 11 creates the protein beta globin in the hemoglobin of red blood cells. This gene is 1600 base long. A single mutation (or base-change) in this gene gives rise to sickle-cell anemia. The figure shows portions of the normal gene and mutated gene. The codon GAG is changed to GTG, which means that the amino acid changes from glutamic acid to valine. This single change in the amino acid chain makes a crucial corner of the 3D protein molecule hydrophobic (water hating), and causes hemoglobin molecules to stick together and create rigid fibres.

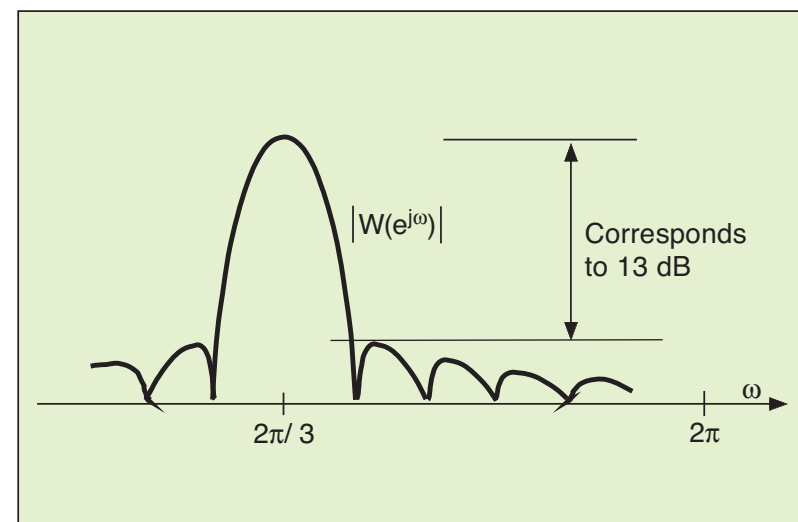

Figure 15. Computation of DFT with a sliding window is equivalent to lowpass digital filtering. The frequency response magnitude is as shown, and offers about $13 \mathrm{~dB}$ stopband attenuation.
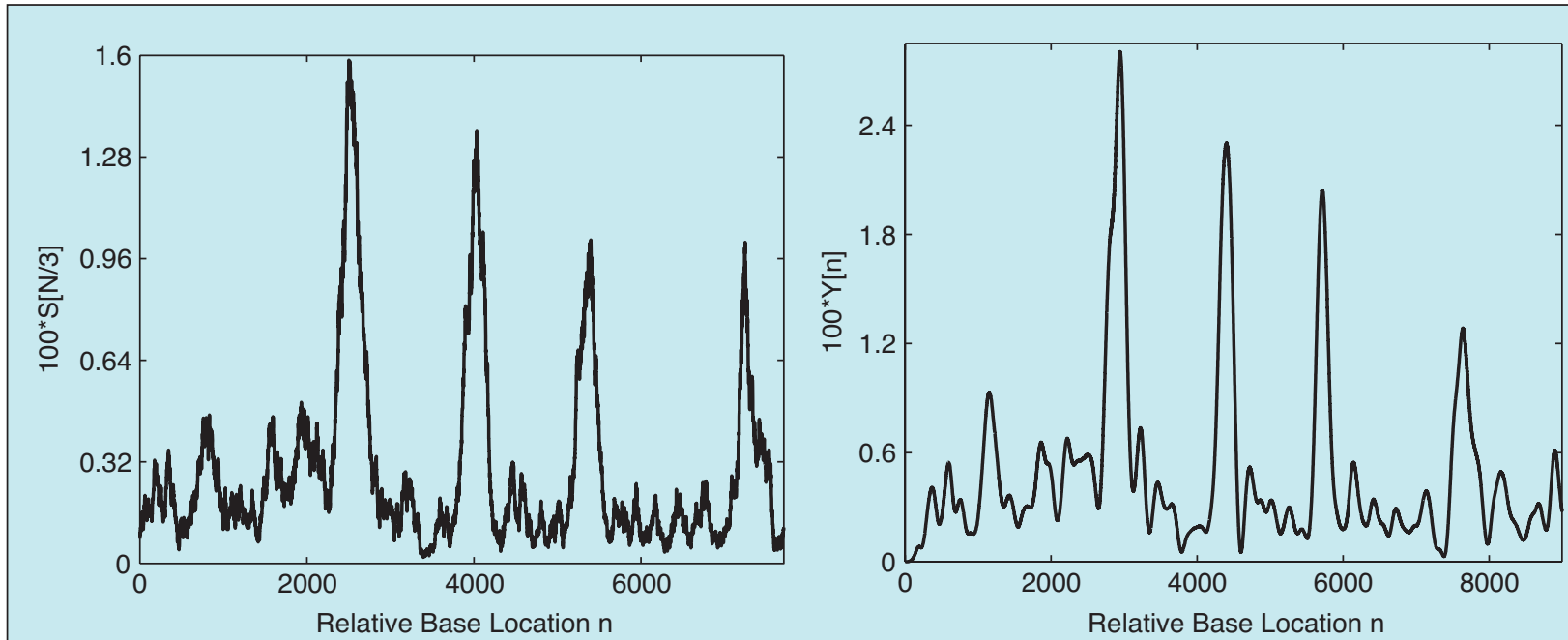

Figure 16. Left plot: the DFT based spectrum S[N/3] for gene F56F11.4 in the C. elegans chromosome III. Right plot: the multistage narrowband bandpass filter output [28] for the same gene. The multistage filter does a very good job of eliminating the $1 / f$ component in the DNA spectrum, and the exon regions are revealed more clearly.

many genes. Long range correlations have been found both in coding and noncoding regions [39]. According to Fourier transform theory, long range correlation implies that the Fourier transform has $1 / f$-behavior in low frequency regions [65].

Another early work on the topic was the 1992 paper by Richard Voss [37] who was perhaps also the first person to define indicator sequences for bases, and calculate the deterministic autocorrelation. For example, letting $x_{A}(n)$ be the indicator for base $A$, the autocorrelation is $r_{A}(k)=\sum_{n} x_{A}(n) x_{A}(n-k)$, and the Fourier transform $S_{A}\left(e^{j \omega}\right)$ of this is the power-spectrum for base $A$. Notice that $S_{A}\left(e^{j \omega}\right)=\left|X_{A}\left(e^{j \omega}\right)\right|^{2}$. Voss analyzed the human Cytomegalovirus strain AD169. The genome length was $N=229,354$. The lowest meaningful frequency ${ }^{5}$ can be regarded as $1 / N$ which is slightly smaller than $0.5 * 10^{-5}$. (Recall that the sample spacing for indicator sequences is normalized to be unity, so the highest frequency $\pi$ corresponds to 0.5.) Voss demonstrated that the power spectrum has power-law or $1 / f^{\beta}$ behavior for each of the four indicator sequences (for appropriate $\beta$ close to unity). Later studies have indicated that such long range correlation is valid even further, extending to several millions of bases [36] (i.e., the $1 / f$ behavior extends to even smaller frequencies). Figure 17 shows the power spectrum $S_{A}\left(e^{j \omega}\right)$ for base $A$ for the first one-million bases of an entire bacterial genome of length about 1.55 million. The

$\overline{5}$ Recall that the sample spacing for indicator sequences is normalized to be unity, so the highest frequency $\pi$ corresponds to 0.5 . 
organism is called Aquifex aeolicus, and its genome can be found in public websites such as the gene bank [29]. There were 0.5 million samples of $S_{A}\left(e^{j \omega}\right)$ in $0 \leq \omega \leq \pi$. The plot shows a slightly smoothed version with a sliding rectangular window of length 33 . Notice that this is a log-log plot and the variations near zero-frequency can be seen clearly. The $1 / f$ behavior continues till very low frequencies, flattening out only as we get really close to zero frequency. Notice also the thin line representing a sharp peak near the right edge of the plot. This corresponds to the peak at $2 \pi / 3$ due to period-3 property in the coding

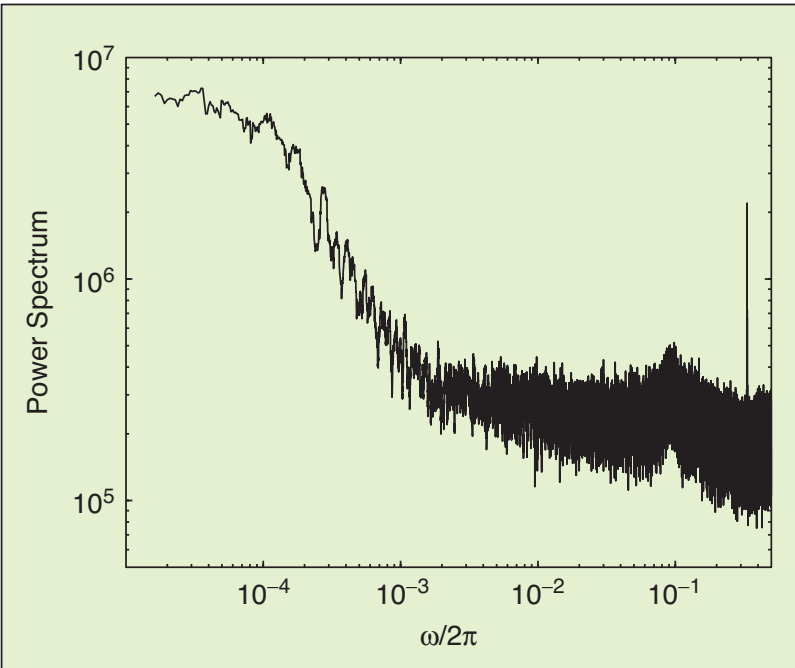

Figure 17. Demonstration of $1 / f$ spectrum. The $1 / f$ behavior extends to very small frequencies indicating very long range correlation. regions. More examples can be found in [36]. Li has written a comprehensive review paper on this topic [33], and has also observed [32] that the $1 / f$ behavior in natural phenomena can be traced to the so-called duplicationmutation model (see Figure 18).

In addition to the overall $1 / f$ behavior of DNA sequences, and the period-3 property in protein coding regions, it has been observed by many authors that DNA molecules also have components of period 10 to 11 (see [31] and references therein). In [31] it is argued that this periodicity can be attributed to an alternation property in protein molecules. This arises from the fact that the hydrophilic and hydrophobic regions (water loving and water hating regions) alternate at a certain rate in the three-dimensional folded form.

\subsection{Fourier Transforms of Protein Sequences}

The ability of a protein to interact selectively with other molecules is of fundamental importance to protein functioning. This ability comes from the very sophisticated 3D shape assumed by a protein depending on its amino acid sequence (e.g., Figure 12, Sec. 5). There are specific sites in the 3D structure called hot spots where certain other molecules can conveniently bind to the protein (see the cartoon demonstration in Figure 19). A protein molecule typically has many functions (many hot spots). Given a collection of proteins, suppose they all have one function in common. Is there a mathematical way to identify this commonality simply by analyzing the amino acid sequence? Yes indeed, based on Fourier techniques [12].

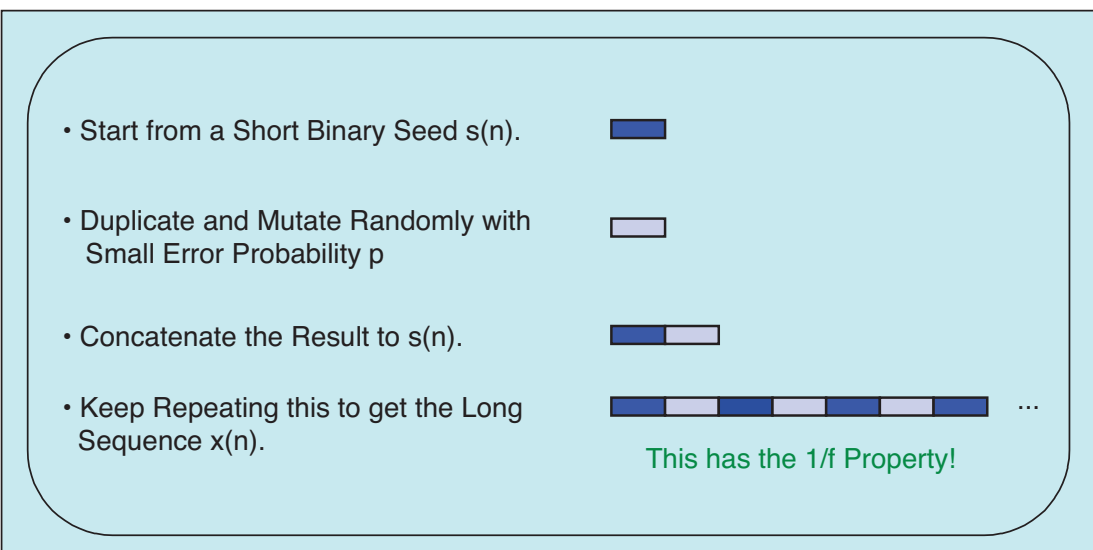

Figure 18. When life started on earth the DNA molecules were short (few thousand bases). As evolution progressed, the molecules went through a lengthening processes which involved duplication and mutation. Imagine we have a character string $s(n)$ of length $L$. Suppose we duplicate it and then make some random changes of certain characters (from the same alphabet), and concatenate the result to the original $s(n)$ to form a sequence that is twice as long. Further repetition of duplication and mutation quickly results in a very long sequence comparable to today's DNA molecules. It can be shown that repeated application of the duplication-mutation process results in $1 / f$ behavior in the spectrum [32].
With each one of the twenty amino acids it is possible to associate a unique nonnegative number called the average electron-ion interaction potential (EIIP). The physical basis for this is explained in [12] and references therein. The EIIP values are shown in Figure 20 and plotted in increasing order in Figure 21. Given a protein, we can associate with it a numerical sequence $x(n)$ such that $x(n)$ is equal to the EIIP value of the $n$th amino acid. The argument $n$ can be regarded as equispaced distance $(\approx 3.8 \AA$ or $0.38 \mathrm{~nm}$, the spacing between amino acids).

Let $X\left(e^{j \omega}\right)=\sum_{n=0}^{N-1} x(n) e^{-j \omega n}$ be the Fourier transform of $x(n)$, where $N$ is the number of the amino acids in the protein. Usually a plot of $\left|X\left(e^{j \omega}\right)\right|$ does not reveal 
much (e.g., see top plots in Figure 22). Now assume that we have a group of proteins. Each protein may have several biological functions but assume that there are some functions that are common to all these proteins. Define the magnitude of the product of the Fourier transforms associated with these proteins as follows: $P\left(e^{j \omega}\right)=\left|X_{1}\left(e^{j \omega}\right) \quad X_{2}\left(e^{j \omega}\right) \ldots X_{M}\left(e^{j \omega}\right)\right|$. It has been observed through extensive experiments that if a group of proteins has only one common function then the product spectrum $P\left(e^{j \omega}\right)$ has one significant peak (bottom plot, Figure 22). This corresponds to the statement that there are common periodic components in the EIIP sequence in the amino acid domain. The physical basis for this arises from the so-called resonant recognition between proteins and their targets [12]. The product $P\left(e^{j \omega}\right)$ has been referred to as the consensus spectrum among the group of proteins used in its definition. The frequency where the peak occurs is

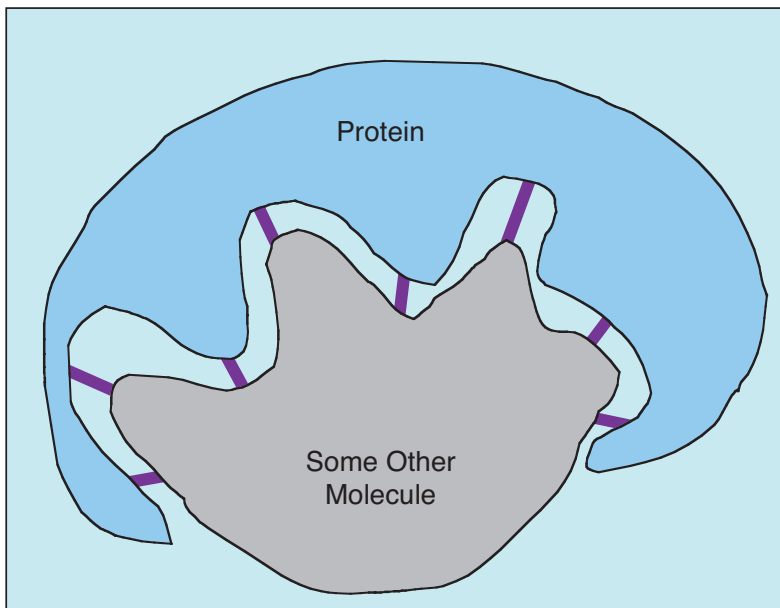

Figure 19. Toy cartoon, showing how the surfaces of certain protein molecules fit like puzzle pieces when they interact.

called the characteristic frequency for the particular protein group. For example, the characteristic frequency is 0.0234 for hemoglobins and 0.3203 for glucagons (frequencies are normalized to be in the range [0, 0.5] as in standard DSP practice). ${ }^{6}$

Assume we have identified that a certain function of a protein is associated with the characteristic frequency $f_{1}$. Is it possible to identify the amino acids that are primarily responsible for that function (i.e., identify

\begin{tabular}{|ccccc|ccclc|}
\hline 1 & $A$ & Ala & Alanine & 0.0373 & 11 & $M$ & Met & Methionine & 0.0823 \\
2 & $C$ & Cys & Cysteine & 0.0829 & 12 & $N$ & Asn & Asparagine & 0.0036 \\
3 & $D$ & Asp & Aspartic acid & 0.1263 & 13 & $P$ & Pro & Proline & 0.0198 \\
4 & $E$ & Glu & Glutamic acid & 0.0058 & 14 & $Q$ & Gln & Glutamine & 0.0761 \\
5 & $F$ & Phe & Phenylalanine & 0.0946 & 15 & $R$ & Arg & Arginine & 0.0959 \\
6 & $G$ & Gly & Glycine & 0.0050 & 16 & S & Ser & Serine & 0.0829 \\
7 & $H$ & His & histidine & 0.0242 & 17 & $T$ & Thr & Threonine & 0.0941 \\
8 & $I$ & Ile & Isoleucine & 0.0000 & 18 & $V$ & Val & Valine & 0.0057 \\
9 & $K$ & Lys & Lysine & 0.0371 & 19 & $W$ & Trp & Tryptophan & 0.0548 \\
10 & $L$ & Leu & Leucine & 0.0000 & 20 & $Y$ & Tyr & Tyrosine & 0.0516 \\
\hline \multicolumn{7}{|l}{ Figure 20. Electron-ion interaction potentials (EIIP) value for the twenty amino acids } \\
[12].
\end{tabular}

the hot spots in the $3 \mathrm{D}$ protein structure which are responsible for one particular function)? This is tricky because the value of a Fourier transform at a given frequency depends on all the time-domain samples. Transforms which offer a local basis such as the wavelet transformation and short time Fourier transformation are more convenient and have been successfully used for this [17], [18]. A detailed study of the use of wavelet transforms in protein structures can be found in the recent paper by Murray et al. [16]. The impact of the use of signal processing tools here could be significant. One advantage of being able to identify a characteristic frequency with a particular functionality is that it is then possible to synthesize artificial amino acid sequences or peptides (short amino acid sequences). These could be potential-

\footnotetext{
${ }^{6}$ Hemoglobins are oxygen carriers in the red blood cells. Glucagons are protein hormones generated in the pancreas, and affect glucose level in blood.
}

ly useful in medicine [17].

\section{Role of Hidden Markov Models}

Markov models are very useful to represent families of sequences with certain specific properties. To explain the idea consider Figure 23(a) which shows a part of a DNA sequence. The base $A$ appears a few times, and it can be followed by an $A, C, T$, or a $G$. Given a long DNA sequence we can count the number of times the base $A$ is followed by, say, a $G$. From this we can estimate the probability that an $A$ is followed by a $G$. If this probability is 0.3 for example, we indicate it as shown in Figure 23(b). The figure also shows examples of probabilities for $A$ of transition to other bases, including itself. The first row of the matrix in Figure 23(c) shows the four probabilities more compactly (notice that their sum is unity). Similarly the probabilities that the base $C$ would transition into the four bases can be estimated, and is shown in the second row of the matrix. This $4 \times 4$ matrix is called a state transition matrix, and is 


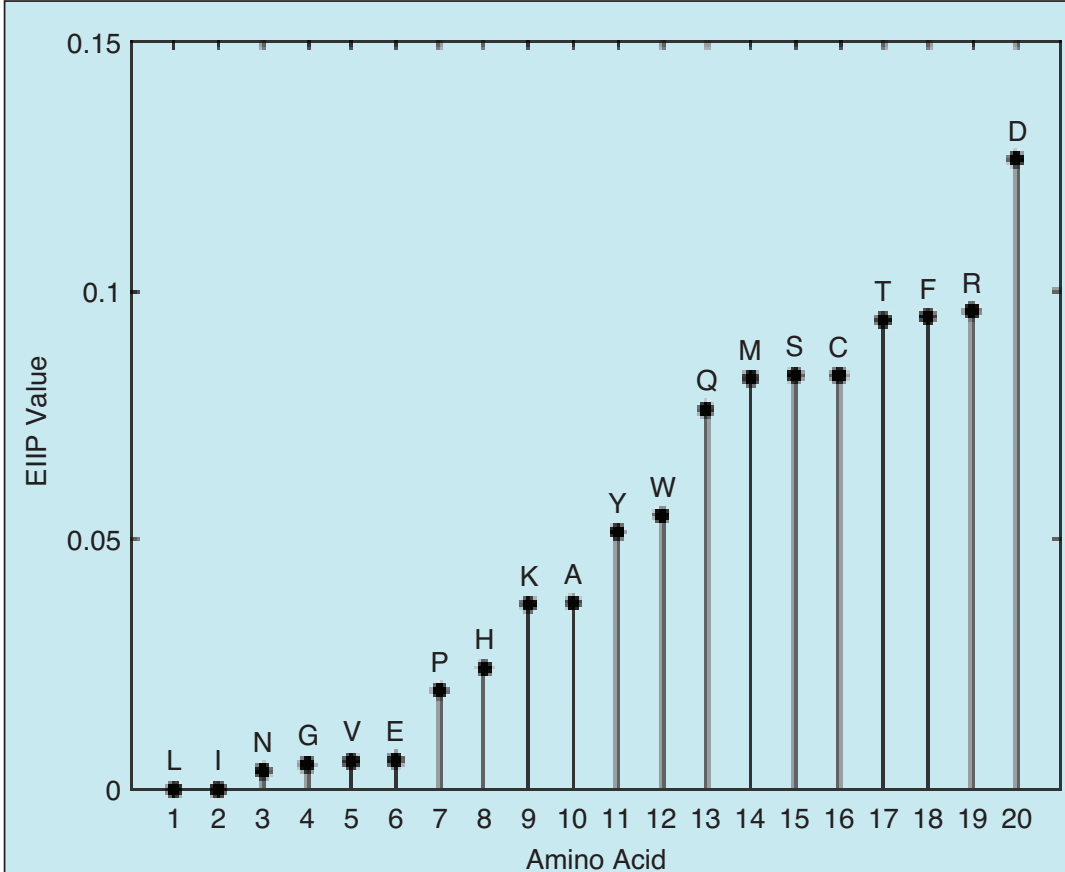

Figure 21. Plot of the electron-ion interaction potential (EIIP) for the twenty amino acids. modification of the Markov model. Thus consider the state diagram shown in Figure 24(a) which shows three states numbered 1,2 , and 3 . The probabilities of transitions from the states are also indicated, resulting in the state transition matrix $\Sigma$ shown in Figure 24(b). When the system is in a particular state, it can output one of four possible symbols, namely $A, T, C$, or $G$, and there is a probability associated with each of these. This is demonstrated in Figure 24(c), and summarized more compactly in the so-called output matrix $\Pi$ shown in Figure 24(d). To give an example of how HMMs might be useful, we can imagine that state 1 corresponds to exons, state 2 to introns, and state 3 to intergenic spaces. In each of these states, the probabilities of transitions between bases could be different. denoted as $\boldsymbol{\Sigma}$. Figure 23(b) is called a Markov model. The four states in this model are $A, C, T$, and $G$. Given a sequence or a set of sequences of "similar kind" (e.g., a long list of exons from several genes) the parameters of the model (the transition probabilities) can readily be estimated. The process of identifying the model parametes is called training the model. In all discussions it is implicitly assumed that the probabilities of transitions are fixed and do not depend on past transitions.

Suppose we are given a Markov model (i.e., $\boldsymbol{\Sigma}$ given). Given an arbitrary state sequence $\mathbf{x}=[x(1)$, $x(2), \ldots, x(L)]$ we can calculate the probability that $\mathbf{x}$ has been generated by our model. This is given by the product

$$
\begin{aligned}
P(\mathbf{x})= & P(x(1)) \times P(x(1) \rightarrow x(2)) \times P(x(2) \rightarrow x(3)) \\
& \times \ldots \times P(x(L-1) \rightarrow x(L))
\end{aligned}
$$

where $P(x(k) \rightarrow x(m))$ is the transition probability for going from $x(k)$ to $x(m)$, and can be found from the matrix $\boldsymbol{\Sigma}$. The usefulness of such computation is as follows: given a number of Markov models ( $\boldsymbol{\Sigma}_{1}$ for introns, $\boldsymbol{\Sigma}_{2}$ for exons, and so forth) and given a sequence $\mathbf{x}$, we can calculate the probabilities that this sequence is generated by any of these models. The model which gives the highest probability is most likely the model which generated the sequence.

A hidden Markov model (HMM) is obtained by a slight
In order to apply the hidden Markov model theory successfully there are three problems that need to be solved in practice [6]. These are listed below along with names of standard algorithms which have been developed for these.

1 Given an HMM (i.e., given the matrices $\boldsymbol{\Sigma}$ and $\boldsymbol{\Pi}$ ) and an output sequence $y(1), y(2), \ldots$, compute the state sequence $x(k)$ which most likely generated it. This is solved by the famous Viterbi's algorithm.

2 Given the HMM and an output sequence $y(1), y(2), \ldots$, compute the probability that the HMM generates this. The forward-backward algorithm solves this.

3 The third problem is training: how should one design the model parameters $\boldsymbol{\Sigma}$ and $\Pi$ such that they are optimal for an application, e.g., to represent exons? The most popular algorithm for this is the expectation maximization algorithm commonly known as the EM algorithm or the BaumWelch algorithm.

Further details on these algorithm can be found in [6]. The theory of HMMs has been applied successfully to gene identification, to identification of special regions of DNA such as CpG islands, and to DNA sequence alignment. There are many good references which explain the use of HMMs in molecular biology. A good start would be to look at [24], [25], [7], and [4], and then proceed to ref- 


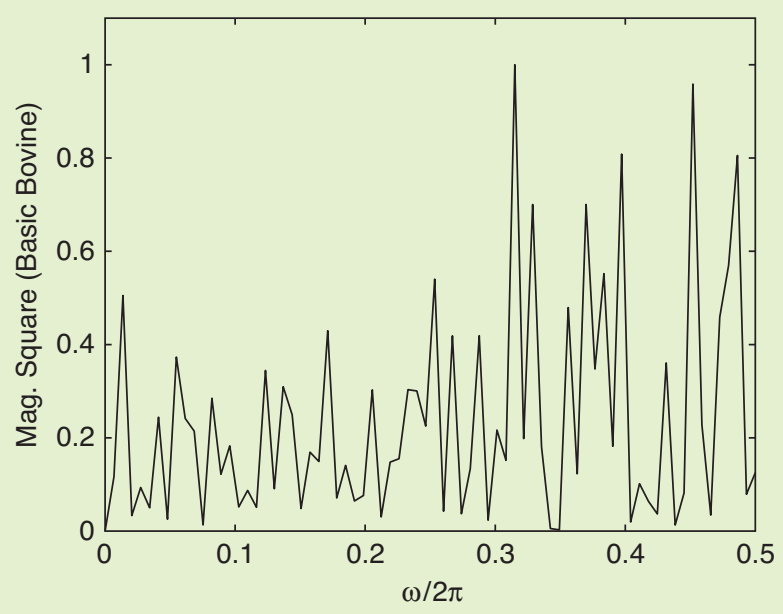

(a)

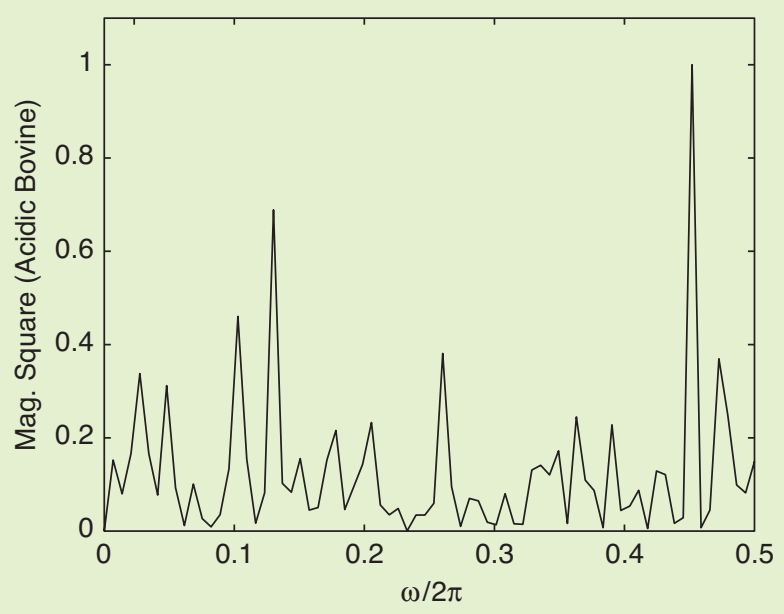

(b)

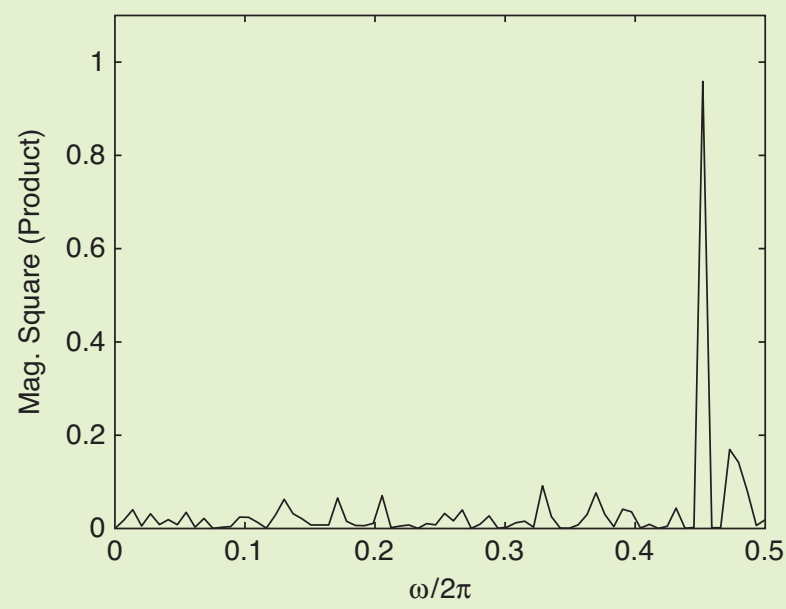

(c)

Figure 22. Magnitude squares of the Fourier transforms of the EIIP sequences for the proteins FGF basic bovine (a) and FGF acidic bovine (b). The product, which represents the square of the consensus spectrum, is plotted in (c) [12].

erences therein. As for basics, there are excellent tutorials and books which explain the theory of Hidden Markov models. The paper by Rabiner in the Proceedings of the IEEE [6] has been widely cited in the molecular biology literature. The books by Rabiner and Juang [66] and by Jelinek [61] give wonderful exposure to the theory and its applications in speech recognition.

\section{Non Coding Genes and ncRNA}

The most common meaning associated with genes during the four decades following the discovery of the double helix was that genes are those parts of the DNA sequence that code for proteins (Sec. 3). But it has become increasingly clear in the last ten years that there are portions of DNA which are transcripted to RNA sequences that do not get translated to proteins. These are called noncoding RNA or ncRNA, and the portions of DNA which generate them are called noncoding genes. Many of these are located in the intergenic space (space between protein coding genes). Indeed ncRNAs have been known for many years, the transfer RNA (tRNA) and ribosomal RNA (rRNA) being classic text-book examples [2]. However, the recognition that there are many different ncRNAs and that noncoding genes play a hereditary role is more recent. The fact that noncoding genes have such tremendous importance has been regarded as a challenge to the central dogma of molecular biology which suggests that genes by definition code for proteins (Sec. 3). So the intergenic space cannot by any means be regarded as "junk DNA" as it used to be once. An excellent place to start reading about noncoding genes is the Scientific American article by Gibbs 
[42]. Papers by Eddy such as the Nature genetics review article [40] are informative as well as insightful.

Perhaps the discovery of the importance of noncoding genes can be traced back to the case of a C. elegans baby that wouldn't grow up. ${ }^{7}$ In an observation made by Ambrose et al. (Dartmouth Medical School, Hanover, $\mathrm{N}$. H) there was a C. elegans baby in the laboratory which grew in size but never came out of the first larva stage (see analogy of a human baby, Figure 25). The scientists were able to trace this to a defective gene. In the healthy worm the function of this gene was to produce

(a) AACTGAGGTACAATTCGATCTC

(b)

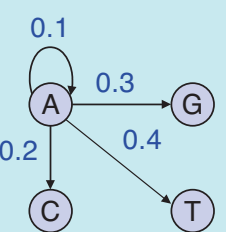

Figure 23. Explaining the basic principle of the Markov model. (a) A sequence of bases, (b) the state diagram showing the transitions from $A$, and (c) an example of the state transition matrix.

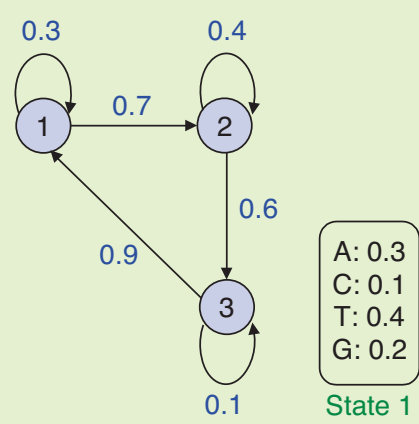

(a)

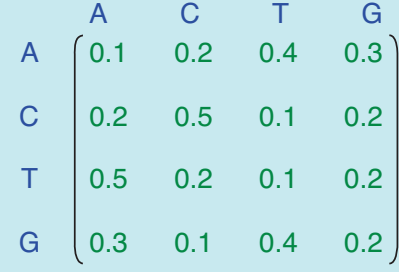

(c)
State Transition Matrix $\Sigma$ a tiny RNA molecule, only 22 bases long. The role of this RNA molecule was to regulate other protein coding genes responsible for normal growth into adult. So this RNA did not get translated into a protein; it was an ncRNA, and functioned all by itself. In the defective C. elegans baby, this particular ncRNA gene in the DNA was mutated, and the ncRNA was not functioning properly, thereby affecting growth functions. This was the first ncRNA recognized (besides tRNA and so forth), and ncRNAs were taken seriously only after this observation. See the short but fascinating account given by John Travis in [49].

Many more ncRNAs have been found in several organisms in the last ten years and their functions identified [40], [48]. It has been conjectured [42] that about fifty percent of the genes in mice generate ncRNAs rather than proteins! C. elegans has more than 200 genes generating micro ncRNAs (tiny ncRNAs about 22 bases long). And the E. coli bacterium has several hundred noncoding genes and about 4200 protein coding genes [40]. Today it is recognized that heriditary information is carried by protein coding genes, noncoding genes and a third layer of information storage called the epigenetic layer [43].

Noncoding genes have created a great deal of excitement in medicine. Other related research not discussed here include the role of double strand RNAs and antisense RNAs in gene silencing. These are called siRNAs (small interfering RNAs) and can be inserted into cells to prevent the expression of hazardous genes. A good starting point for the interested reader is the series of Scientific American articles [43]-[45]. The discovery of noncoding genes apparently solves a longheld puzzle in biology. It has been known that the number of protein coding genes never scales in proportion to the size of the organism [42]. For example, worms have only twice as many protein-coding genes as bacteria. Humans have only thrice as much (about 35,000). And the rice plant has more genes than humans! But if the number of noncoding genes is counted, it seems that the total number of genes does scale well with the complexity of organisms [42].

\subsection{Identifying Noncoding Genes}

In Sec. 6.1 we explained that there are many ways to identify protein coding genes in DNA sequences. These genes have a period-3 component due to codon bias which is usually quite strong. For more precise identification one can use hidden Markov models as explained in [24]. Computational identification of noncoding genes is much more difficult. These genes could be very small (sometimes no longer than 22 bases), do not exhibit the period3 property, and do not have start and stop codons.

\footnotetext{
${ }^{7}$ C. Elegans is a worm or nematode used extensively in biological studies. It grows into an adult with exactly 959 cells.
} 

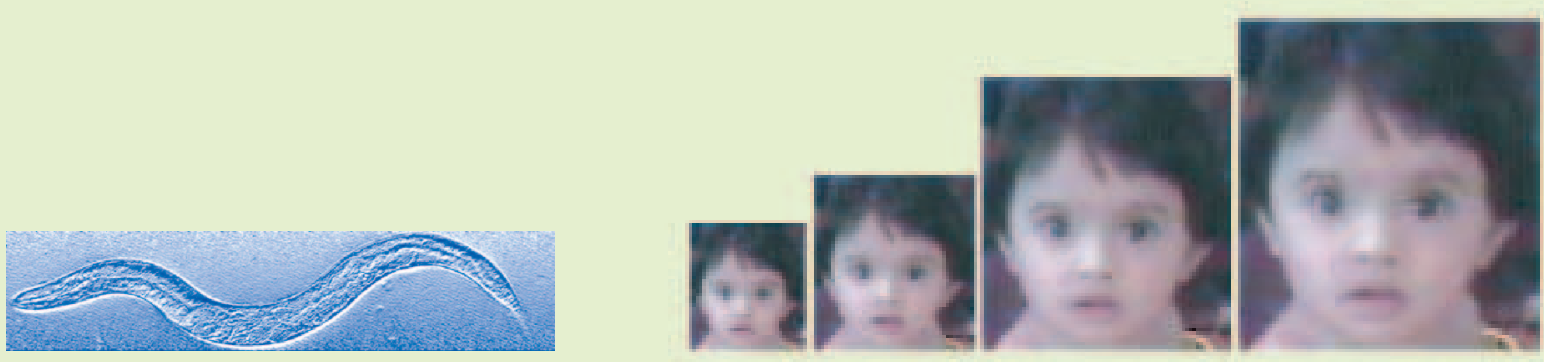

Figure 25. Left: the C. elegans worm, magnified many times. Right: if a human baby grew in size but not in features, that would be analogous to the $C$. elegans story which lead to the discovery of the importance of ncRNA genes. See text.

Conclusions could often be wrong; there are case histories where certain genes, originally thought to be ncRNA genes, were later found to encode tiny proteins [40].

It has been noticed that noncoding genes and ncRNAs function by virtue of their secondary structure which we explain next. Consider Figure 26 which shows an ncRNA in E. coli bacteria. Notice that even though it is a single stranded molecule (like most RNAs are), there are long stretches of bases in one part which are complementary to stretches in other parts (recall here that $A$ pairs up with $U$ and $C$ with $G$ ). This complementarity forces the RNA to fold into shapes which are not only beautiful, but in fact are crucial to their biological functioning. Many of the RNAs can act as enzymes primarily by virtue of this folded shape. RNA enzymes are called ribozymes, so they are not confused with normal enzymes which are proteins. Some computational biologists have suggested that noncoding genes in the DNA sequences can be identified simply by looking for subsequences which have secondary structure [47].

We will return to this later but briefly mention another approach called comparative genomics which has been reasonably successful. The idea behind comparative genomics is that if two or more species have a common stretch of DNA, then it is probably doing something important. Otherwise nature would not have conserved it for millions of years. So these stretches would have to be either protein coding genes or noncoding genes. If they do not pass standard tests for protein coding genes they are likely to be noncoding genes. In this way it is possible to accumulate a list of potential noncoding genes in a given species and then check them by other biological means. Comparative study of DNA sequences is not as simple as it appears to be on first sight because the sequences being compared come from various species, and "identical regions" can still differ due to mutations, insertions, and deletions of bases through millions of years of evolution. For example consider the following four sequences:

where $x$ denotes that the base could be any one of the four. Inspection reveals that there are many common patterns here. However, a direct comparison base by base would lead a computer to conclude that these are

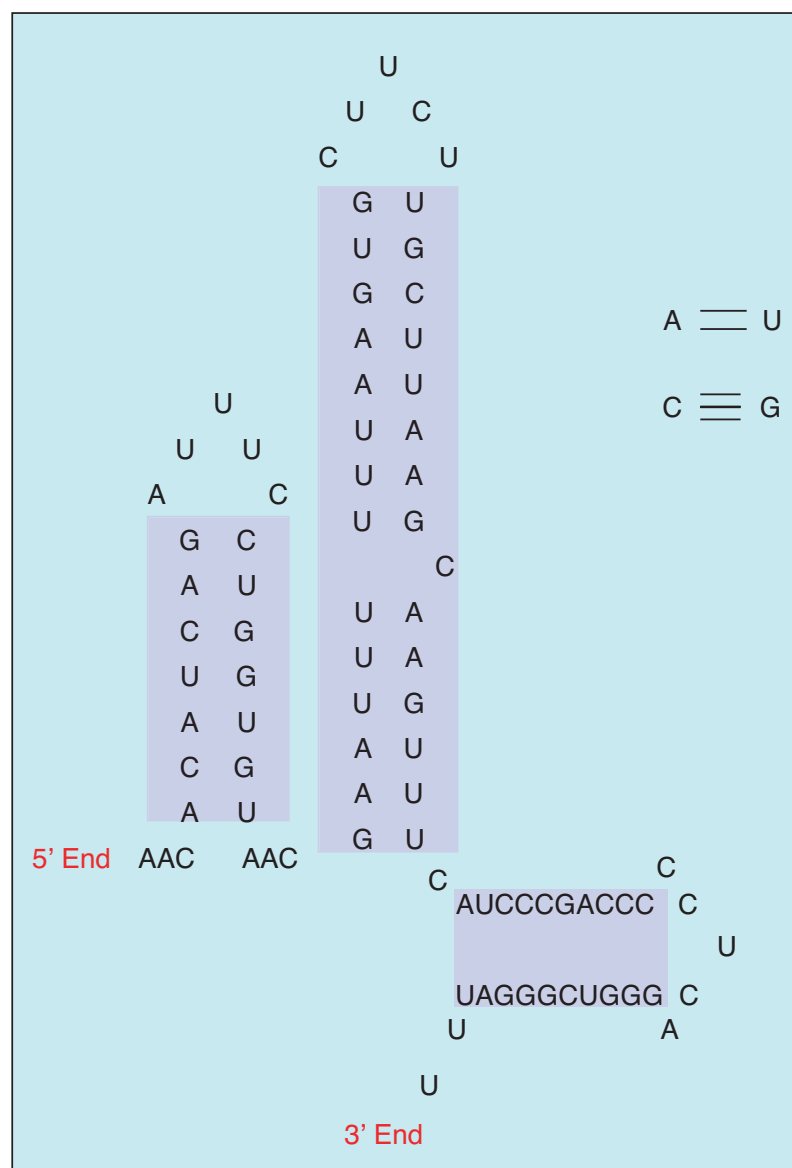

Figure 26. The picture shows a dsrA RNA in E. coli. Notice the secondary structure created by base pairing in blue shaded areas. Functionality of ncRNAs depends mostly on their secondary structure. 
not identical sequences at all. There are patterns which are common but with slight mutations; there are unequal gaps between similar patterns; and the "identi- ture rather than the exact sequence of base pairs. For example the two sequences shown below would fold the same way.

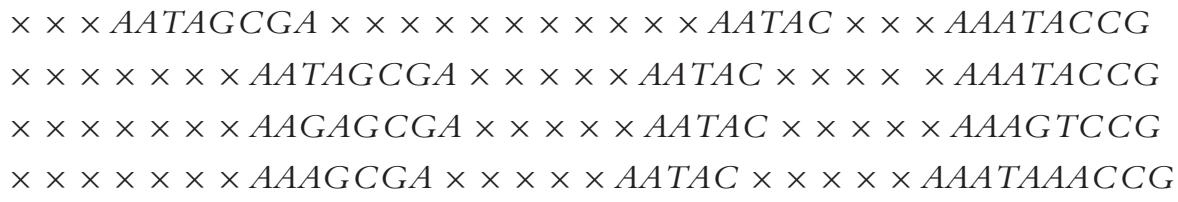

cal parts" often do not even have identical lengths!

The task of comparing such sequences is nontrivial science. It comes under the topic of sequence alignment. Computational biologists have developed many methods for this and, in fact, assign scores to the degree of similarity between sequences. Markov models have been used for this application. Many wonderful details can be found in the book by Durbin et al. [4]. In a study by the National Human Genome Research Institute (NHGRI) the human genome has been compared with many others such as cow's, dog's, pig's, and rat's. It has been found that there were over 150 common regions in the intergenic space! Many potential ncRNA sequences have been listed in this way and later confirmed by other means. The method of comparative genomics to identify ncRNAs does not work perfectly yet, but has been quite useful.

\subsection{Identifying Secondary Structure}

A few words on the identification of secondary structures directly without comparative genomics. Consider Figure 27(a) which shows a DNA sequence with two short subsequences AATC and GATT buried in it. These subsequences are separated by many bases. If we reverse the first subsequence we get CTAA which is complementary to the second sequence. So the subsequences can be regarded as two halves of a palindrome (i.e., a symmetric sequence like $x y z p q p z y x){ }^{8}$ The sequence can therefore fold as shown in Figure 27(b) and remain stable in that configuration because of the $A-T$ and $C-G$ bondings. If an ncRNA is generated from such a DNA segment it would therefore fold as shown. In practice, the matching subsequences do not match exactly, they may be separated by an arbitrary number of bases, and furthermore there may be more than one matching pair. The secondary structure can therefore be quite complicated. All of these features can be clearly seen in the example of ncRNA shown in Figure 26.

The biological functioning of the ncRNA depends primarily on the way it folds, that is, on the secondary struc-

\footnotetext{
${ }^{8}$ Not exactly a palindrome because of the complement operation, but we shall refrain from inventing a new word for that.
}

$$
\begin{aligned}
& \times \times \times A A T C \times \times \times \times \times \times \times \times \times \times G A T T \times \times \times \\
& \times \times \times A G T A \times \times \times \times \times \times \times \times \times T A C T \times \times \times
\end{aligned}
$$

Computational identification of ncRNA genes is therefore closely related to the identification of buried patterns such as palindromes in a long arbitrary sequence (a few thousand or million bases). This is quite a challenging problem. One of the theoretical bottlenecks is that hidden Markov models which worked so well for identification of protein coding genes do not work anymore as explained next.

\subsection{Grammars}

In the language of computer science, a grammar is a set of rules which can be repeatedly applied to obtain sequences of letters from an alphabet. The set of all sequences that can be generated by a grammar is called the language generated by that grammar. In the early 1950s, Noam Chomsky (a phenomenal computational linguist from MIT) classified grammars into four types called regular grammars, context free grammars, context sensitive grammars, and unrestricted grammars. The relation between these grammars is depicted in Figure 28.

Regular grammars have the most restricted production rules and therefore generate a restricted class of languages. Context free grammars allow a wider class of production rules and generate a broader class of languages. For example suppose the "language" is the set of all palindromes. Then there is no regular grammar to generate these, but there does exist a context free grammar. ${ }^{9}$

We now give a very brief overview of grammars. Good references to this topic include [60] and [62]. A regular grammar allows production rules of the form $W \rightarrow a W$ and $W \rightarrow a$, where $W$ is a nonterminal symbol (i.e., we can make further substitutions for it) and $a$ is a terminal symbol. An alphabet is specified from which the terminals are taken. Consider the example of a regular grammar with the following three production rules, where $A, C$, and $T$ are the terminals: 9 True, we can find a regular grammar which generates palindromes
among other possible sequences. But we cannot find a regular grammar which generates only palindromes. 


$$
\begin{aligned}
& W \rightarrow A W, \quad W \rightarrow T W \quad W \rightarrow C W, \\
& W \rightarrow A, \quad W \rightarrow T, \quad \text { and } \quad W \rightarrow C .
\end{aligned}
$$

Here is an example of a string generated by this grammar by application of the rules in arbitrary order:

$$
W \rightarrow A W \rightarrow A A W \rightarrow A A C W \rightarrow A A C T W \rightarrow A A C T T
$$

The language generated by this grammar is the string of all DNA sequences with the base $G$ missing.

A context free grammar allows production rules of the form $W \rightarrow \alpha$ where $W$ is a nonterminal and $\alpha$ is a string of terminals and nonterminals. A grammar defined by the following production rules is an example. Here $A, C, G$, and $T$ are the terminals.

$$
\begin{aligned}
& W \rightarrow A W A, \quad W \rightarrow C W C, \\
& W \rightarrow T W T \quad W \rightarrow G W G \text { and } W \rightarrow \epsilon
\end{aligned}
$$

where $\epsilon$ represents the null string (i.e., nothing). Here is an example of a string generated by this grammar:

$$
W \rightarrow A W A \rightarrow A T W T A \rightarrow A T C W C T A \rightarrow A T C C T A
$$

In the last step $W$ has been replaced with the null terminal character. Notice that the resulting string is a palindrome. The preceding grammar generates the palindromes language.

If the production rules in a grammar are used with a certain probability attached to each rule, it is called a stochastic grammar. There is a result in the theory of computations which says that stochastic regular grammars are identical to hidden Markov models. That is, if a class of strings can be generated by a stochastic regular grammar then there exists an HMM which generates this class, and vice versa. Since regular grammars cannot generate palindrome languages we cannot therefore build HMMs that represent noncoding genes. We cannot therefore use HMM theory to identify noncodig genes buried in long DNA sequences. Stochastic context free grammars, abbreviated as SCFGs, have been used for this purpose and a great deal of detail can be found in [4] and references therein. Figure 29 summarizes some of these discussions.

Recall from Sec. 7 that in order to apply the HMM theory successfully there are three problems that need to be solved, and there exist standard algorithms for this, namely Viterbi's algorithm, forward-backward algorithm, and the EM algorithm. For the case of context free grammars there are similar algorithms but they have much higher complexity [4]. The importance of fast procedures

\section{Sequence with a Palindrome Buried in it xxxAATC $x x x x x x x x x x x x x x x x x x x x x x x$ GATT $x x x x x x x x x x x$}

(a)
Folded

Sequence $x x x$ AATC $x x x x x x x x x x x x x x x x_{x}$ |||| $x x x x x x x x x x x$ TTAG $x x x x x x x x x x x x x x x x^{x}$

(b)
Figure 27. (a) Example of a palindrome-like pattern buried in DNA, and (b) the natural way for this sequence to fold.

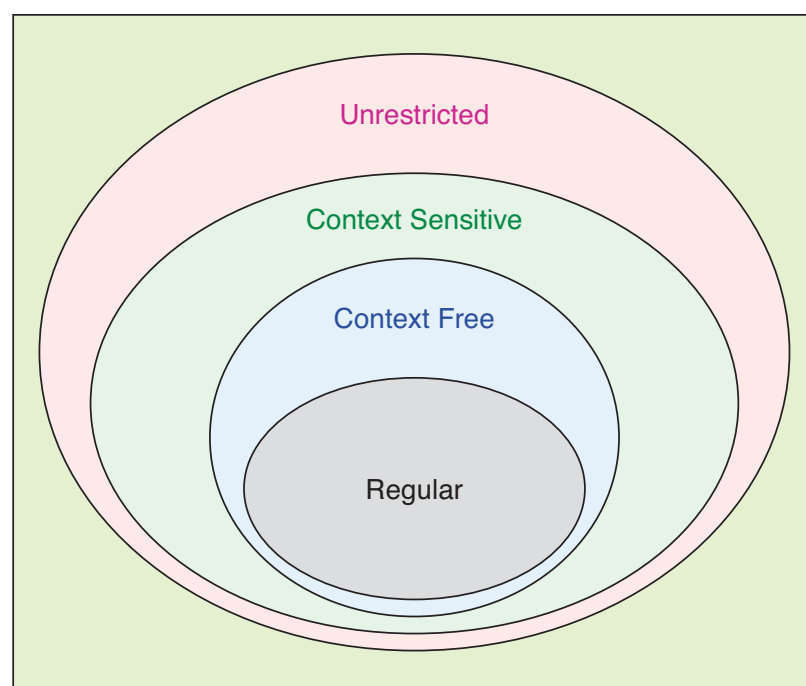

Figure 28. Chomsky's hierarchy of grammars for generating languages.

for these arises because of the fact that DNA sequences are very long even for "small" organisms. Computational biologists are therefore interested in developing faster algorithms for the above problems. Recently Yoon and Vaidyanathan have introduced a class of hidden Markov models called context sensitive HMMs [50] which appear to be promising for this application while at the same time offering significantly lower complexity.

Finally, even context sensitive languages have had some applications in this context. An example of a language that can be recognized by such grammars but not by context free grammars is the so-called copy language [4] which can sometimes be useful in describing secondary structures.

\section{Other Areas}

In the past few sections a number of interesting areas were discussed but many were also left out for want of space. One of these is DNA computation. The enormous capabilities of the cell (base-pairing, gene-protein feedback) can be used to perform miraculously difficult computational tasks. A starting point for the reader would be 
the article by Adleman in 1998 in the Scientific American [59]. Another area we did not discuss is DNA sequencing. Many signal processing aspects are involved here, and a flavor can be obtained by reading [14] and [22]. An informal discussion of some other areas is given here with appropriate pointers to literature.

\subsection{DNA Microarrays}

An entire issue of Nature Genetics was dedicated to the topic of DNA microarrays in 1999. The reader should see [53] and other articles therein for an excellent introduction. A good overview also appeared in the IEEE Spectrum a few years ago [57], so we will be brief. DNA microarrays are typically grown on a piece of glass or silicon substrate chemically primed so that the molecules $A, C, T$ and $G$ stick to specific sites. It is possible to raise towers of base sequences about 100 bases long, using photolithography as shown in Figure 30. In this way an entire gene can be

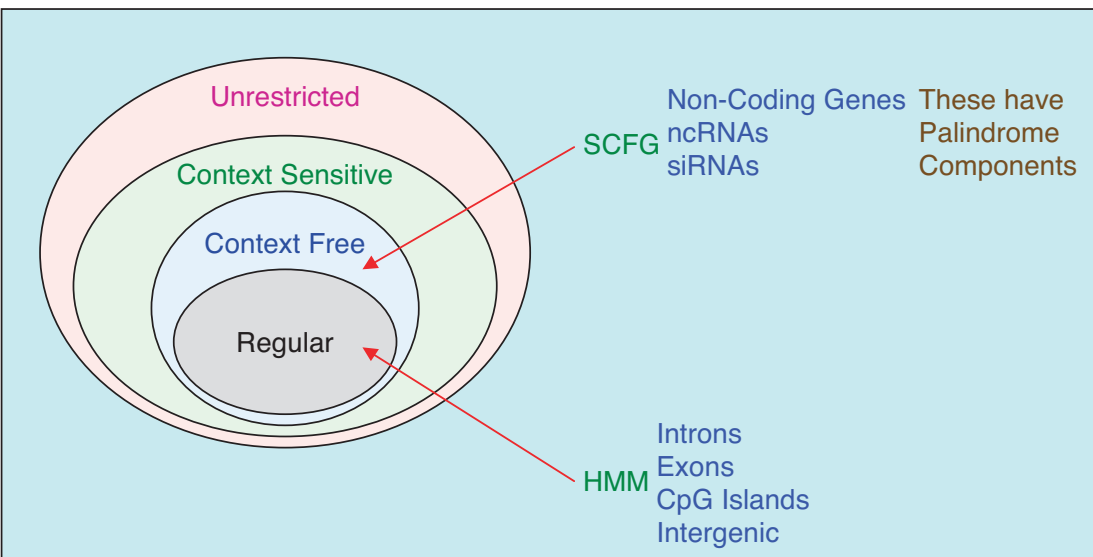

Figure 29. Application of grammars in computational biology. Regular grammars (stochastic hidden Markov models) are useful for identifying protein coding genes whereas stochastic context free grammars (SCFGs) are necessary to identify noncoding genes.

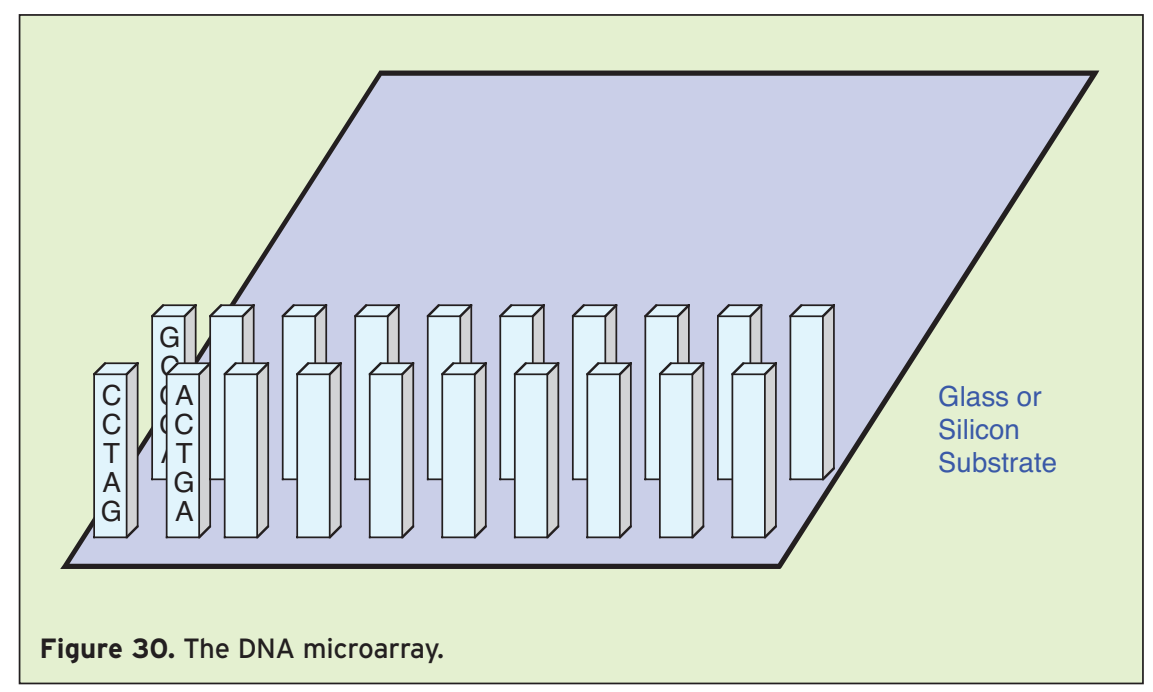

"grown" on a few towers. Several genes can therefore be captured onto a single DNA microarray chip.

These chips can be used to observe the expression levels of different genes in the cell as explained in Figure 31 . The real advantage here is that we can measure the levels of several genes simultaneously, and as a function of time (e.g., cell cycle) and so forth. This gives an enormous advantage to biologists who wish to study the dependency of gene expressions on various factors. An example is the 1999 experiment at MIT [57] where Affymetrix chips containing 6800 human genes were used to analyze the expression of genes in cancer cells from two types of blood cancer (acute myeloid leukemia and lymphoblastic leukemia). Standard pathology examination failed to distinguish the two types but the arrays showed a set of 50 genes that have different activity levels in the two cancers. Many examples can be found in the papers published in Nature genetics, Jan. 1999, and papers such as [51]. DNA microarrays have serious application in drug design [55], antiterrorism [54], and many other related areas.

The Affymetrix series started with a modest 1000 genes on chip in 1998. Today, nearly all of the protein coding genes in humans (about 35,000) have gone into a single chip (Affymetrix Inc. and Agilent Technologies announced this in 2003). There are some interesting signal processing issues involved in the interpretation of data recorded on a DNA array. Some examples can be found in [58] and [51].

\subsection{The Gene-Protein Feedback Loop}

We know that genes guide the generation of proteins. But proteins to a large extent also control which genes are expressed and to what extent. In short, proteins can switch genes on and off. The gene-protein feedback loop is what make different cells look and function differently. Cell function depends on a gene-protein network interconnected in a highly complex manner.

The first hint that proteins in cells might be influencing gene 
expression came from Francois Jacob and Jacques Monad in Paris, around 1960. The E. coli bacteria uses lactose sugar and breaks it into simpler sugars (galactose and glucose) using the enzyme beta galactosidase. When lactose is absent in the bacterial medium the E. coli cell does not produce this enzyme. Otherwise it does! Jacob and Monad suggested that this switching ability is due to the presence of a repressor molecule. In the late 60s Walter Gilbert and Benno Müller-Hill (from Harvard) found the molecule. The repressors were proteins and this was the first proof that there is a closed loop (feedback) system. In recent years, the closed loop relation has been described with some success using linear first order coupled differential equations called Langevin equations [19], and this has been found to be useful in systematic analysis of uncertainties (or "noise") in gene circuits. A fascinating account of information pro-

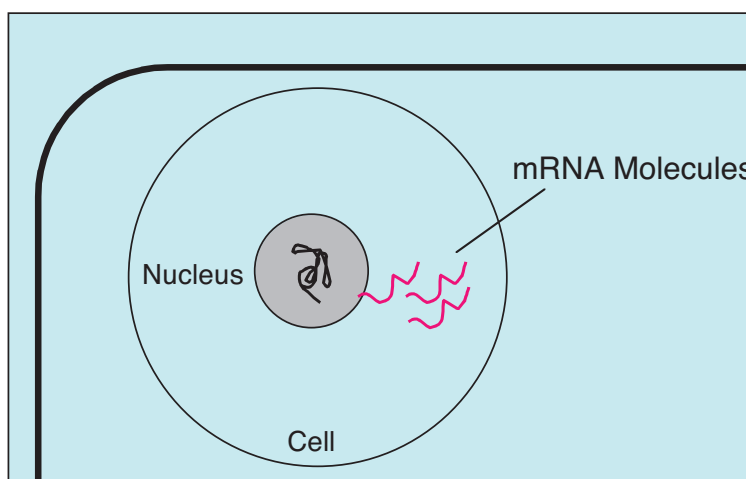

(a)

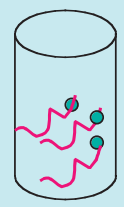

(b)

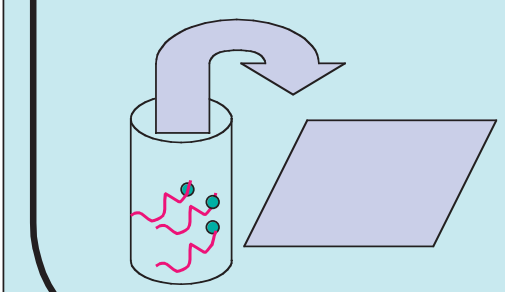

(c)

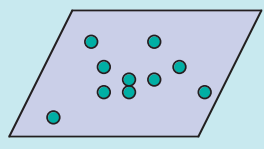

(d)

Figure 31. Measuring gene expression levels using DNA microarrays. (a) When a gene is expressed, mRNA molecules are released from the nucleus. (b) These mRNA molecules are collected, turned into DNA, and tagged with florescent dyes. (c) This genecocktail is poured onto the gene micro-array. The tagged molecules stick to the portions of genes on the array which are complementary to them. (d) If the array is now illuminated with a light source, then the tags glow and one can see which genes are expressed. Strength of the glow indicates the amount of gene expression. cessing in genetic circuits can be

found in the May 2004 IEEE paper by Simpson et al. [20].

\subsection{Relation to RNA World}

If proteins are generated by genes and genes are in turn controlled by proteins, then which came first? This is similar to asking whether the chicken or egg came first. The fact that ncRNA molecules can perform many of the functions of proteins (Sec. 8) answers this question to some extent. There is a theory called the RNA-world theory which suggests that the earliest form of life on earth was based entirely on RNA molecules. Some of these RNA molecules carry genetic information (like genes in DNA), whereas some act as catalysts. ${ }^{10}$ The article by Orgel in the Scientific American [46] traces the origin of this theory and gives an account of some laboratory experiments which demonstrate the feasibility of the RNA-world theory.

\section{Concluding Remarks}

In this article we have attempted to share the excitement of molecular biology from the point of view of the scien-

\footnotetext{
10 RNA catalysts are called ribozymes rather than enzymes; the latter name is reserved for protein catalysts.
}

tist with a signal processing and circuits background. We conclude with the sentiment that genomics, and more generally molecular biology have taken a very interesting turn for all of us. For those who did not like biology because of the wet smelly labs, there is good news. Molecular biology today involves signal processing, computer science, mathematics, and informatics, all coming together beautifully!

\section{Acknowledgements}

It is my pleasure to thank Professors A. Antoniou, L. Bruton, and M. N. S. Swamy, for strongly encouraging me to write this article. I am grateful to Professor M. Ogorzalek for all his support and help during the production of this article. Many of the Fourier transform and digital filtering plots in this paper were prepared by Mr. Byung-Jun Yoon at the California Institute of Technology.

\section{References}

We have tried to categorize the papers into subtopics. Many papers can easily belong to more than one category. So please do not overlook any of these. The selection here is by no means complete but based on my personal taste. Perhaps a good list to start with, to teach from, to make reading-assignments from, and so forth.

The paper which started it all ... 
[1] J. D. Watson and F. H. C. Crick, "A structure for DNA," Nature, p. 737 April 1953.

\section{Books and Tutorials}

[2] B. Alberts, D. Bray, A. Johnson, J. Lewis, M. Raff, K. Roberts, and P. Walter, Essential Cell Biology, Garland Publishing Inc., New York, 1998. [3] D. Anastassiou, "Genomic signal processing," IEEE Signal Processing Magazine, pp. 8-20, July 2001.

[4] R. Durbin, S. R. Eddy, A. Krogh, and G. Mitchison, Biological Sequence Analysis, Cambridge Univ. Press, 1998.

[5] Proc. of IEEE special issues, Dec. 2000 (Genomic Engineering), Nov. 2002 (Bioinformatics, part 1: Advances and Challenges), and Dec. 2002 (Bioinformatics, part 2: Genomics and Proteomics Engineering).

[6] L. R. Rabiner, "A tutorial on hidden Markov models and selected applications in speech recognition," Proc. of the IEEE, vol. 77, no. 2, pp. 257-286, Feb. 1989.

[7] S. L. Salzberg, D. B. Searls, and S. Kasif, Computational Methods in Molecular Biology, Elsevier, 1998.

[8] P. P. Vaidyanathan and B-J. Yoon, "The role of signal processing concepts in genomics and proteomics," Journal of the Franklin Institute, vol. 341, pp. 111-135, 2004

[9] J. D. Watson, The Double Helix, Simon and Schuster, NY, 1968.

[10] J. D. Watson, Genes, Girls, and Gamow, Vintage books, Random House, Inc., NY, 2001.

[11] J. D. Watson (with Andrew Berry) DNA: The Secret of Life, Alfred A Knopf, NY, 2003.

\section{Signal-processing flavor (DNA/Protein)}

[12] I. Cosic, "Macromolecular bioactivity: Is it resonant interaction between macromolecules?-theory and applications," IEEE. Trans. Biomedical Engr., vol. 41, no. 12, pp. 1101-1114, Dec. 1994.

[13] S. W. Davies, M. Eizenman, and S. Pasupathy, "Optimal structure for automatic processing of DNA sequences," IEEE Trans. on Biomedical Engr., vol. 46, no. 9, pp. 1044-1056, Sept. 1999.

[14] W. Huang, D. R. Fuhrmann, D. G. Politte, L. J. Thomas, and D. J. States, "Filter matrix estimation in automated DNA sequencing," IEEE Trans. on Biomedical Engr, vol. 45, no. 4, pp. 422-428, April 1998.

[15] R. Koradi, M. Billeter, and K. Wthrich, "MOLMOL: A program for display and analysis of macromolecular structures," J. of Mol. Graphics, vol. 14, pp. 51-55, 1996

[16] K.B. Murray, D. Gorse, and J. M. Thornton, "Wavelet transforms for the characterization and detection of repeating motifs," J. Molecular Biology, vol. 316, pp. 341-363, 2002.

[17] E. Pirogova, Q. Fang, M. Akay, and I. Cosic, "Investigation of the structural and functional relationships of oncogene proteins," Proc. of the IEEE, vol. 90, no. 12, pp. 1859-1867, Dec. 2002.

[18] P. Ramachandran, A. Antoniou, and P. P. Vaidyanathan, "Identification and location of hot spots in proteins using the short-time Fourier transform," presented at IEEE Asilomar Conference on Signals, Systems, and Computers, Monterey, CA, Nov. 2004.

[19] M. L. Simpson, C. D. Cox, and G. S. Sayler, "Frequency domain analysis of noise in autoregulated gene circuits," Proc. of the Nat. Academy of Sci., vol. 100, no. 8, pp. 4551-4556, April 15, 2003.

[20] M. L. Simpson, C. D. Cox, G. D. Peterson, and G. S. Sayler, "Engineering in the biological substrate: Information processing in genetic circuits," Proc. of the IEEE, vol. 92, no. 5, pp. 848-863, May 2004.

[21] D. Sussillo, A. Kundaje, and D. Anastassiou, "Spectrogram analysis of genomes," Eurasip J. of Applied Signal Processing, vol. 2003, no. 4, Dec. 2003.

[22] X-P. Zhang and D. Allison, "Iterative deconvolution for automatic base scaling of the DNA electrophoresis time series," presented at Workshop on Genomic Sig. Proc. and Stat., Raleigh, NC, Oct. 2002.

\section{Gene prediction}

[23] J. W. Fickett, "The gene prediction problem: An overview for developers," Computers Chem., vol. 20, no. 1, pp. 103-118, 1996.

[24] A. Krogh, I. Saira Mian, and D. Haussler, "A hidden Markov model that finds genes in E. Coli DNA," Nucleic Acids Research, vol. 22, pp. $4768-4778,1994$.

[25] S. L. Salzberg, A. L. Delcher, S. Kasif, and O. White, "Microbial gene identification using interpolated Markov models," Nucleic Acids Research, vol. 26, no. 2, pp. 544-548, 1998.

[26] S. Tiwari, S. Ramachandran, A. Bhattacharya, S. Bhattacharya, and R. Ramaswamy, "Prediction of probable genes by Fourier analysis of genomic sequences," CABIOS, vol. 13, no. 3, pp. 263-270, 1997. [27] P. P. Vaidyanathan and B-J. Yoon, "Gene and exon prediction using allpass-based filters," presented at Workshop on Genomic Sig. Proc. and Stat., Raleigh, NC, Oct. 2002.

[28] P. P. Vaidyanathan and B-J. Yoon, "Digital filters for gene prediction applications," presented at IEEE Asilomar Conference on Signals, Systems, and Computers, Monterey, CA, Nov. 2002.

[29] National Center for Biotechnology Information, Available: http://www.ncbi.nlm.nih.gov/.

\section{Long range correlations, $1 / f$ behavior, statistics}

[30] H. Hausdorff and C.-K. Peng, "Multiscaled randomness: A possible source of $1 / f$ noise in biology," Physical Review E, vol. 54, no. 2, pp. 2154-2157, August 1996.

[31] H. Herzel, E. N. Trifonov, O. Weiss, and I. Gro $\beta$ e, "Interpreting correlations in biosequences," Physica A, vol. 249, pp. 449-459, 1998. [32] W. Li, "Expansion-modification systems: A model for spatial $1 / f$ spectra," Physical Review A, The American Physical Society, vol. 43, no. 10 , pp. 5240-5260, May 1991.

[33] W. Li, "The study of correlation structures of DNA sequences: A critical review," Computers Chem., vol. 21, no. 4, pp. 257-271, 1997.

[34] C. K. Peng, S. V. Buldyrev, A. L. Goldberger, S. Havlin, F. Sciortino, M. Simons, and H.E. Stanley, "Long-range correlations in nucleotide sequences," Nature, vol. 356, pp. 168-170, March 1992.

[35] E. N. Trifonov and J. L. Sussman, "The pitch of chromatin DNA is reflected in its nucleotide sequence," Proc. of the Nat. Acad. Sci., USA, vol. 77, pp. 3816-3820, 1980.

[36] M. de Sousa Vieira, "Statistics of DNA sequences: A low-frequency analysis," Physical Review E, The American Physical Society, vol. 60 no. 5, pp. 5932-5937, Nov. 1999.

[37] R. F. Voss, "Evolution of long-range fractal correlations and $1 / f$ noise in DNA base sequences," Physical Review Letters, vol. 68, no. 25, pp. 3805-3808, June 1992.

[38] G. W. Wornell, "A Karhunen-Loeve-like expansion for $1 / f$ processes via wavelets," IEEE Trans. on Information Theory, vol. 36, no. 4, pp. 859-861, July 1990

[39] Z-G. Yu, V. V. Anh, and B. Wang, "Correlation property of length sequences based on global structure of the complete genome," Physical review E, The American Physical Society, vol. 63, pp. 011903-1-011903-8, 2000 .

\section{Noncoding genes, ncRNA}

[40] S. R. Eddy, "Noncoding RNA genes and the modern RNA world," Nature Reviews, GENETICS, vol. 2, pp. 919-929, Dec. 2001.

[41] S. R. Eddy, "Computational genomics of noncoding RNA genes," Cell, vol. 109, pp. 137-140, April 2002.

[42] W. W. Gibbs, "The unseen Genome: Gems among junk," Scientific American, pp. 48-53, Nov. 2003.

[43] W. W. Gibbs, "The unseen Genome: Beyond DNA," Scientific American, pp. 108-113, Dec. 2003.

[44] W. W. Gibbs, "Synthetic life," Scientific American, pp. 75-81, May 2004.

[45] N. C. Lau and D. P. Bartel, "Censors of the genome," Scientific American, pp. 35-41, August 2003.

[46] L. E. Orgel, "The origin of life on earth," Scientific American, pp. 77-83, Oct. 1994.

[47] E. Rivas, R. J. Klein, T. A. Jones, and S. R. Eddy, "Computational identification of noncoding RNAs in E. Coli," Current Biology, vol. 11, pp. 1369-1373, Sept. 2001.

[48] G. Storz, "An expanding universe of noncoding RNAs," Science, vol. 296, pp. 1260-1263, May 2002.

[49] J. Travis, "Biological Dark matter," Science News Online, Jan. 12 2002. Available: www.sciencenews.org/articles /20020112/bob9.asp.

[50] B-J. Yoon and P. P. Vaidyanathan, "HMM with auxiliary memory: A new tool for modelling RNA secondary structures," presented at IEEE Asilomar Conference on Signals, Systems, and Computers, Monterey, CA, Nov. 2004.

\section{DNA microarrays}

[51] O. Alter, P. O. Brown, and D. Botstein, "Singular value decomposition for genome-wide expression data processing and modeling," Proc. of the Natl. Acad. of Sci., USA, vol. 97, no. 18, pp. 10101-10106, Aug. 2000.

[52] O. Alter, P. O. Brown, and D. Botstein, "Generalized singular value 
decomposition for comparative analysis of genome-scale expression data sets of two different organisms," Proc. of the Natl. Acad. of Sci., USA, vol. 100, no. 6, pp. 3351-3356, March 2003.

[53] P. O. Brown and D. Botstein, "Exploring the new world of the genome with DNA microarrays," Nature Genetics Supplement, vol. 21, pp. 33-37, Jan. 1999.

[54] R. Casagrande, "Technology against terror," Scientific American, pp. 82-87, Oct. 2002.

[55] C. Debouck and P. N. Goodfellow, "DNA microarrays in drug discovery and development," Nature Genetics Supplement, vol. 21, pp. 48-50, Jan. 1999.

[56] E. S. Lander, "Array of hope," Nature Genetics Supplement, vol. 21, pp. 3-4 Jan. 1999.

[57] S. K. Moore, "Making chips to probe genes," IEEE Spectrum, vol. 38 , no. 3, pp. 54-60, March 2001.

[58] Y. Wang, J. Lu, R. Lee, Z. Gu, and R. Clarke, "Iterative normalization of cDNA microarray data," IEEE Trans. Info. Tech. in Biomed., vol. 6 no. 1, pp. 29-37, March 2002.

Other related general references ... .

[59] L. M. Adleman "Computing with DNA," Scientific American, pp. 54-61, Aug. 1998

[60] A. V. Aho, J. E. Hopcroft, and J. D. Ullman, The Design and Analysis of Computer Algorithms, Addison Wesley Publ. Co., Reading, MA, 1974.

[61] F. Jelinek, Statistical Methods for Speech Recognition, The MIT Press, Cambridge, MA, 2001.

[62] H. R. Lewis and C. H. Papadimitriou, Elements of the Theory of Computation, Prentice Hall, Inc., Englewood Cliffs, NJ, 1981.

[63] Y. Neuvo, C.-Y. Dong, and S. K. Mitra, "Interpolated finite impulse response filters," IEEE Trans. on ASSP, pp. 563-570, June 1984.

[64] A. V. Oppenheim and R. W. Schafer, Discrete-time Signal Processing, Prentice Hall, Inc., NJ, 1999.

[65] A. Papoulis, Systems and Transforms with Applications in Optics, McGraw Hill, 1968.

[66] L. R. Rabiner and B-H. Juang, Fundamentals of Speech Recognition, Prentice Hall, Inc., Englewood Cliffs, NJ, 1993.

[67] P. P. Vaidyanathan, Multirate Systems and Filter Banks, Prentice Hall, Inc., NJ, 1993.

P. P. Vaidyanathan (S'80-M'83-SM'88-F'91) was born in Calcutta, India on Oct. 16,1954. He received the B.Sc. (Hons.) degree in physics and the B.Tech. and M.Tech. degrees in radiophysics and electronics, all from the University of Calcutta, India, in 1974, 1977 and 1979, respectively, and the Ph.D. degree in electrical and computer engineering from the University of California at Santa Barbara in 1982. He was a post doctoral fellow at the University of California, Santa Barbara from Sept. 1982 to March 1983. In March 1983 he joined the electrical engineering department of the California Institute of Technology as an Assistant Professor, and since 1993 has been Professor of electrical engineering there. His main research interests are in digital signal processing, multirate systems, wavelet transforms and signal processing for digital communications.

Dr. Vaidyanathan served as Vice-Chairman of the Technical Program committee for the 1983 IEEE International symposium on Circuits and Systems, and as the Technical Program Chairman for the 1992 IEEE International symposium on Circuits and Systems. He was an Associate editor for the IEEE Transactions on Circuits and Systems for the period 1985-1987, and is currently an associate editor for the journal IEEE Signal Processing letters, and a consulting editor for the journal
Applied and computational harmonic analysis. He has been a guest editor in 1998 for special issues of the IEEE Trans. on Signal Processing and the IEEE Trans. on Circuits and Systems II, on the topics of filter banks, wavelets and subband coders. Dr. Vaidyanathan has authored a number of papers in IEEE journals, and is the author of the book Multirate systems and filter banks. He has written several chapters for various signal processing handbooks. He was a recipient of the Award for excellence in teaching at the California Institute of Technology for the years 1983-1984, 1992-93 and 1993-94.

He also received the NSF's Presidential Young Investigator award in 1986. In 1989 he received the IEEE ASSP Senior Award for his paper on multirate perfectreconstruction filter banks. In 1990 he was recipient of the S. K. Mitra Memorial Award from the Institute of Electronics and Telecommunications Engineers, India, for his joint paper in the IETE journal.

He was also the coauthor of a paper on linear-phase perfect reconstruction filter banks in the IEEE SP Transactions, for which the first author (Truong Nguyen) received the Young outstanding author award in 1993. Dr. Vaidyanathan was elected Fellow of the IEEE in 1991. He received the 1995 F. E. Terman Award of the American Society for Engineering Education, sponsored by Hewlett Packard Co., for his contributions to engineering education, especially the book Multirate systems and filter banks published by Prentice Hall in 1993. He has given several plenary talks including at the IEEE ISCAS-04, Sampta-01, Eusipco-98, SPCOM-95, and Asilomar-88 conferences on signal processing. He has been chosen a distinguished lecturer for the IEEE Signal Processing Society for the year 1996-97.

In 1999 he was chosen to receive the IEEE CAS Society's Golden Jubilee Medal. He is a recipient of the IEEE Signal Processing Society's Technical Achievement Award for the year 2002 .

\section{Author's address:}

P. P. Vaidyanathan

Dept. Electrical Engr., 136-93,

California Institute of Technology,

Pasadena, CA 91125.

Ph:(626) 3954681

Email: ppvnath@systems.caltech.edu 